\title{
Characterization of Piezoelectric Wafer Active Sensors
}

\author{
Victor Giurgiutiu* and Andrei N. Zagrai \\ University of South Carolina, Department of Mechanical Engineering, Columbia, SC 29208
}

\begin{abstract}
In the beginning, the classical one-dimensional analysis of piezoelectric active sensors is reviewed. The complete derivation for a free-free sensor is then extended to cover the cases of clamped and elastically constrained sensors. An analytical model based on structural vibration theory and theory of piezoelectricity was developed and used to predict the electro-mechanical (E/M) impedance response, as it would be measured at the piezoelectric active sensor's terminals. The model considers one-dimensional structures and accounts for both axial and flexural vibrations. The numerical analysis was performed and supported by experimental results. Experiments were conducted on simple beam specimens to support of the theoretical investigation, and on thin gauge aluminum plates to illustrate the method's potential. It was shown that E/M impedance spectrum recorded by the piezoelectric active sensor accurately represents the mechanical response of a structure. It was further proved that the response of the structure is not modified by the presence of the sensor, thus validating the sensor's non-invasive characteristics. The sensor calibration procedure is outlined and statistical analysis was presented. It was found that PZT active sensors have stable and repeatable characteristics not only in as-received condition, but also while mounted on 1-D or 2-D host structure. It is shown that such sensors, of negligible mass, can be permanently applied to the structure creating a non-intrusive sensor array adequate for on-line automatic structural identification and health monitoring.
\end{abstract}

\section{INTRODUCTION}

The advent of commercially available low-cost piezoceramics has opened new opportunities for dynamic structural identification using embedded active sensors. Embedded active sensors are small piezoelectric (PZT) ceramic wafers that can be permanently attached to the structure (Figure 1). Through their intrinsic electromechanical (E/M) coupling, these piezoelectric wafers act as both sensors and actuators. In addition, their frequency bandwidth is orders of magnitude larger than that of conventional modal analysis equipment. They can form sensor and actuator arrays that permit effective modal identification in a wide frequency band.

Crawley and Luis (1987) proposed the use of piezoceramic wafers as elements of intelligent structures. Dimitriadis et al. (1991) and D’Cruz (1993) used piezoelectric wafers for structural excitation. Zhou et al. (1996) performed experiments in which a PZT wafer produced the excitation, while a laser velocimeter picked up the vibration response. Several investigators (Collins et al. (1992), Clark et al. (1993)) and others used piezopolymer films for vibration sensing. Banks (1996) describes experiments in which the PZT wafer was used initially for excitation, and then for sensing the free decay response. Wang and Chen (2000) used a PZT wafer to excite the structure and an array of PVDF film sensors to pick up the forced vibration response to generate the frequencies and mode shapes through multi-point signal processing. Liang et al. (1994) performed the coupled E/M analysis of adaptive systems driven by a surface-attached piezoelectric wafer. The aim of the analysis was to determine the actuator power consumption and system energy transfer. A 1-degree of freedom (1-DOF) analysis was performed, and the electrical admittance, as measured at the terminals of the PZT wafer attached to the structure, was expressed as:

$$
Y(\omega)=i \omega C\left(1-\kappa_{31}^{2} \frac{Z_{s t r}(\omega)}{Z_{s t r}(\omega)+Z_{A}(\omega)}\right)
$$

where $C$ is the electrical capacitance of the PZT active sensor, $Z_{s t r}(\omega)$ is the 1-DOF structural impedance as seen by the sensor, and $Z_{A}(\omega)$ is the sensor impedance. A 1DOF numerical example, using a quasi-static sensor impedance formulation, was used to show that the E/M admittance response accurately reflects the system dynamic response. At coupled-system resonance, the real part of the E/M admittance was shown to have a distinct peak. However, due to the additional stiffness contributed by the PZT wafer, the system natural frequency shifted from $500 \mathrm{~Hz}$ (without PZT wafer) to $580 \mathrm{~Hz}$ (with PZT wafer). Experimental curve-fitting results were also presented. No modeling of the structural substrate was included, and no prediction of $Z_{s t r}(\omega)$ for a multi-DOF structure was presented. This work was continued and extended by Sun et al. (1994) who used the half-power bandwidth method to accurately determine the natural frequency and damping values. Mode shape extraction methods, using the self and across admittance of multiple sensors, were explored. Experiments were performed on aluminum beams at frequencies up to $7 \mathrm{kHz}$. These two papers were the first to conceptualize that the $\mathrm{E} / \mathrm{M}$ admittance as seen at the sensor terminals reflects the coupled-system dynamics, and that an embedded PZT wafer could be used as structural-identification sensor. However, no theoretical modeling of the $E / M$

* Author to whom correspondence should be addressed, Email victorg@sc.edu 
impedance/admittance response for comparison with

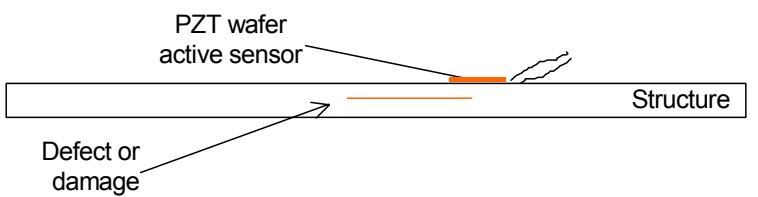

Figure 1 PZT wafer acting as active sensor to detect and monitor structural damage

Nor were investigated the issues of sensor calibration and consistency. Subsequently, several authors reported the use of the E/M impedance method for structural health monitoring, whereby the admittance or impedance frequency spectra of pristine and damaged structures were compared (Chaudhry et al., 1994, 1995; Ayres et al., 1996; Giurgiutiu et al., 1997, 1999; Park, Cudney, and Inman, 2000)). The method has been shown to be especially effective at ultrasonic frequencies, where it can properly capture the changes in local dynamics due to incipient structural damage. (Such changes are too small to affect the global dynamics and hence cannot be readily detected by conventional low-frequency vibration methods). The method is direct and easy to implement, since the only required equipment is the electrical impedance analyzer. In spite of extensive experimental effort investigation of the electromechanical impedance method, little theoretical modeling work has been reported to date. Park, Cudney, and Inman (2000) showed that "there is little analytical work done about the vibration modes of complex structures at ultrasonic frequencies" and hence restricted their analysis to axial vibrations. In addition, only the structural displacement response was predicted. A few other investigators have attempted to model structures with piezoelectric active sensors (Wang and Rogers; 1991; Zhou et al., 1996; Esteban, 1996), but none have derived explicit expressions for predicting the $\mathrm{E} / \mathrm{M}$ admittance and impedance, as it would be measured by an electrical impedance analyzer connected to the embedded active sensor terminals during the structural identification process. Such a derivation is necessary to permit complete understanding of the phenomenon and to allow critical comparison with the abundant experimental data.

In response to this need, this work sets forth to present a step-by-step derivation of the piezoelectric sensor dynamics for various boundary conditions and its interaction with the host structure. The classical approach for free vibrations of piezoelectric sensor was reviewed and numerical simulations are presented. An expression for elastically constrained sensor, containing the full sensor dynamics, will be derived. This will open the path towards full inclusion of the structural dynamics and the development a fully coupled sensor-structure model. experimental data was attempted.

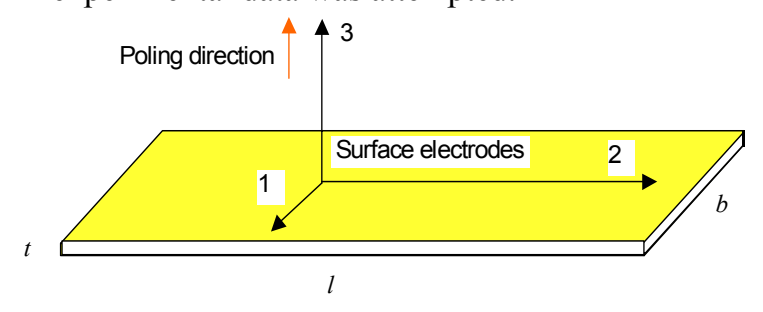

Figure 2 Schematic of a PZT active sensor

Analytical expressions and numerical results for the E/M admittance and impedance as seen at sensor terminals will be produced. These numerical results will be directly compared with experimental measurements performed at the piezoelectric active sensor terminals during the structural identification process. In our derivation, the limitations of the quasi-static sensor approximation adopted by previous investigators are lifted. Exact analytical expressions are being used for structural modeling of simultaneous axial and flexural vibrations. Free-free boundary conditions for the host structure that can unequivocally be implemented during experimental testing (though more difficult to model) are being used.

Another important aspect covered in this work is that of sensor calibration, reliability and repeatability. These aspects are essential for the qualification of new sensor concepts. The intrinsic properties of the piezoelectric active sensors in the as received condition (single wafers), and after adhesive attachment to the 1-D and 2-D structures were statistically studied. Good consistency and repeatability was found throughout our work.

\section{MODELING OF A SINGLE PZT ACTIVE SENSOR}

The dynamics of a single PZT active sensor is first considered. The modeling of a single PZT sensor is useful for two reasons (a) understanding the electromechanical coupling between the mechanical vibration response and the complex electrical response of the sensor; and (b) sensor screening and quality control prior to installation on the structure. Various boundary conditions (free; clamped; elastically constrained) will be analyzed. The free boundary condition is a classical case (Onoe and Jumoji; 1967; Pugachev et al., 1984; IEEE Std. 176, 1987; Parton and Kudryavtsev, 1988; Ikeda, 1996), which allows us to introduce the notations to the reader and set the stage for the other two cases. The clamped case is important as the antithesis of the free case. Whereas the elastically constrained case represents a generic situation, which asymptotically tends to each of the previous two cases as the constraint becomes vanishingly soft, or infinitely stiff, respectively. The elastically constrained case opens the path to the analysis of the complete sensor-structure dynamics, as covered in later sections of this paper. 


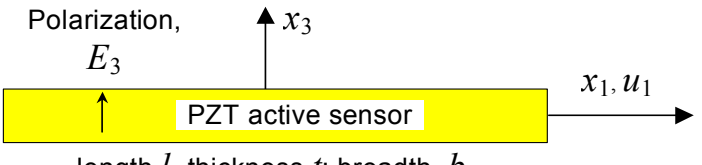

length $l$, thickness $t$; breadth, $b$.

Figure 3 PZT wafer active sensor schematic.

Consider a PZT wafer of length $l$, breadth $b$, and thickness $t$, undergoing displacements, $u_{1}, u_{2}, u_{3}$ induced by the electric polarization field, $E_{3}$ (Figure 3 ). The electric field is produced by the application of a harmonic voltage $V(t)=$ $\hat{V} \mathrm{e}^{\mathrm{i} \omega t}$ between the top and bottom surface electrodes. The resulting electric field, $E=V / t$, is assumed spatially uniform $\left(\partial E / \partial x_{1}=\partial E / \partial x_{2}=\partial E / \partial x_{3}=0\right)$. The constitutive equations of the PZT material are:

$$
\begin{aligned}
& S_{1}=s_{11}^{E} T_{1}+s_{12}^{E} T_{2}+s_{13}^{E} T_{3}+d_{31} E_{3} . \\
& S_{2}=s_{21}^{E} T_{1}+s_{22}^{E} T_{2}+s_{23}^{E} T_{3}+d_{32} E_{3} \\
& S_{3}=s_{31}^{E} T_{1}+s_{32}^{E} T_{2}+s_{33}^{E} T_{3}+d_{33} E_{3} \\
& D_{3}=d_{31} T_{1}+d_{32} T_{2}+d_{33} T_{3}+\varepsilon_{3}^{T} E_{3} .
\end{aligned}
$$

where $S_{i}$ is the strain, $T_{i}$ is the stress, $D_{3}$ is the electrical displacement (charge per unit area), $s_{i j}^{E}$ is the mechanical compliance at zero field, $\varepsilon_{3}^{T}$ is the dielectric constant at zero stress, $d_{3 i}$ is the induced strain coefficient, i.e., mechanical strain per unit electric field.

\section{Longitudinal Vibrations of a Free Active Sensor}

To simplify the analysis, the length, breadth, and thickness are assumed to have widely separated values $(t<<b<l)$ such that the length, breadth, and thickness motions are practically uncoupled. For longitudinal vibration, we write:

$$
\begin{aligned}
& S_{1}=s_{11}^{E} T_{1}+d_{31} E_{3} . \\
& D_{3}=d_{31} T_{1}+\varepsilon_{3}^{T} E_{3} .
\end{aligned}
$$

Using Newton's law of motion, $T_{1}^{\prime}=\rho \ddot{u}_{1}$, and the straindisplacement relation, $S_{1}=u_{1}^{\prime}$, Equation (3) yields the axial waves equation:

$$
\ddot{u}_{1}=c^{2} u_{1}^{\prime \prime}
$$

where ()$^{\prime}=\partial() / \partial t$, and ()$^{\prime}=\partial() / \partial x$, while $c^{2}=1 / \rho s_{11}^{E}$ is the wave speed. The general solution of Equation (4) is:

$$
u_{1}(x, t)=\hat{u}_{1}(x) e^{i \omega t}, \quad \hat{u}_{1}(x)=\left(C_{1} \sin \gamma x+C_{2} \cos \gamma x\right)
$$

The variable $\gamma=\omega / c$ is the wave number (Graff, 1975), and (^) signifies the harmonic motion amplitude. The constants $C_{1}$ and $C_{2}$ are to be determined from the boundary conditions. For a free PZT active sensor, stressfree boundary conditions apply at both ends, i.e., $T\left(-\frac{1}{2} l\right)$

$=T\left(\frac{1}{2} l\right)=0$. Equation (3) gives the conditions $\hat{u}^{\prime}\left(-\frac{1}{2} l\right)=\hat{u}^{\prime}\left(\frac{1}{2} l\right)=d_{31} \hat{E}_{3}$. Substitution of Equation (4) yields:

$$
\begin{aligned}
& \gamma\left(C_{1} \cos \frac{1}{2} \gamma l-C_{2} \sin \frac{1}{2} \gamma l\right)=d_{31} \hat{E}_{3} \\
& \gamma\left(C_{1} \cos \frac{1}{2} \gamma l+C_{2} \sin \frac{1}{2} \gamma l\right)=d_{31} \hat{E}_{3}
\end{aligned}
$$

The solution is:

$$
\hat{u}_{1}(x)=\frac{1}{2} u_{I S A} \frac{\sin \gamma x}{\frac{1}{2} \gamma l \cos \frac{1}{2} \gamma l}
$$

This result is consistent with Ikeda (1996), with the only difference of the notation $u_{I S A}=d_{31} \hat{E}_{3} \cdot l$. The poles of Equation (8), correspond to frequency values where the mechanical response to electrical excitation becomes unbounded, i.e., electromechanical resonance. For pure mechanical response (Inman, 1996, pp. 322), resonance occurs at $\gamma l=n \pi, n=0,1,2, \ldots$. The even multiples of $\pi$ correspond to symmetric modes of vibration, while the odd values correspond to anti-symmetric modes. However, the condition for Equation (8) to have unbound values and produce electromechanical resonance is $\frac{1}{2} \gamma l=(2 m+1) \frac{\pi}{2}$

$m=0,1,2, \ldots$. This means that only anti-symmetric vibration modes, corresponding to odd multiples of $\pi$, are amiable to electromechanical resonance. The physical explanation for this phenomenon lies in the fact that the free boundary conditions (6) and (7) imply that the mode shape derivatives have same values at both ends, and this can only happen if the vibration modes shapes are antisymmetric.

Equation (3) can be re-expressed as:

$$
D_{3}=\frac{d_{31}}{s_{11}}\left(u_{1}^{\prime}-d_{31} E_{3}\right)+\varepsilon_{33}^{T} E_{3}=\varepsilon_{33}^{T} E_{3}\left[1+\kappa_{31}^{2}\left(\frac{u_{1}^{\prime}}{d_{31} E_{3}}-1\right)\right]
$$

where $\kappa_{13}^{2}=d_{31}^{2} /\left(s_{11} \varepsilon_{33}\right)$ is the electromechanical coupling factor (IEEE Std. 176 1987). Integration of Equation (9) yields the charge:

$$
Q=\int_{-\frac{l}{2}}^{+\frac{l}{2}} \int_{-\frac{b}{2}}^{+\frac{b}{2}} D_{3} d x d y=\varepsilon_{33}^{T} \frac{b l}{t} V\left[1+\kappa_{31}^{2}\left(\left.\frac{1}{l} \frac{1}{d_{31} E_{3}} u_{1}\right|_{-\frac{l}{2}} ^{+\frac{l}{2}}-1\right)\right]
$$


where $\varepsilon_{33}^{T} b l / t=C$ is the conventional capacitance of the piezoelectric wafer. For harmonic motion, $\hat{I}=\mathrm{i} \omega \cdot \hat{Q}$. Recall the expressions $Y=\hat{I} / \hat{V}$ and $Z=Y^{1}$ for the electric admittance and impedance, respectively. Hence, Equation (10) yields

$$
Y=\mathrm{i} \omega \cdot C\left[1+\kappa_{31}^{2}\left(\frac{\tan \frac{1}{2} \gamma l}{\frac{1}{2} \gamma l}-1\right)\right]
$$

This result agrees with Ikeda (1996). To facilitate comparison with future results, we write it in the form:

$$
\begin{aligned}
& Y=\mathrm{i} \omega \cdot C\left[1-\kappa_{31}^{2}\left(1-\frac{1}{\varphi \cot \varphi}\right)\right] \\
& Z=\frac{1}{\mathrm{i} \omega \cdot C}\left[1-\kappa_{31}^{2}\left(1-\frac{1}{\varphi \cot \varphi}\right)\right]^{-1}
\end{aligned}
$$

where $\varphi=\frac{1}{2} \gamma l$. As for any electrically reactive device, the admittance is purely imaginary. The admittance poles, $\varphi_{Y}=(2 m+1) \frac{\pi}{2}, m=0,1,2, \ldots$ correspond to the electromechanical resonance. At the admittance poles, $\hat{I} \rightarrow \infty$. Mathematically, the admittance follows the behavior of the $1 / \cot \varphi$ function, which goes to $+\infty$, and then suddenly jumps to $-\infty$. Also of interest are the admittance zeros (impedance poles), which are solutions of the equation $\varphi_{Z} \cot \varphi_{Z}=-\kappa_{31}^{2} /\left(1-\kappa_{31}^{2}\right)$. At these values $I$ $=0$. Table 1 shows the admittance and impedance poles calculate for $\kappa_{31}=0.36$. The numerical values of the admittance poles, $\varphi_{Y}$, and impedance poles, $\varphi_{Z}$, differ significantly only for the first few modes. By the fourth mode, the difference between them drops below $0.1 \%$.

Equation (12) can be used to predict the admittance and impedance frequency response. To this purpose, we note that $\omega=2 \pi f$ and $\varphi=\frac{1}{2} l \omega / c$. As the excitation frequency varies, and resonance and anti-resonance frequencies are encountered, the admittance and impedance go through $+\infty$ to $-\infty$ transitions. Outside resonance, the admittance follows the linear function $i \omega C$, while the impedance follows the inverse function $1 /(i \omega C)$.

Table 1 Admittance and impedance poles for $\kappa_{31}=0.36$

\begin{tabular}{llllll}
\hline $\begin{array}{l}\text { Admittance } \\
\text { poles, } \varphi_{Y}\end{array}$ & $\pi / 2$ & $3 \pi / 2$ & $5 \pi / 2$ & $7 \pi / 2$ & $9 \pi / 2$ \\
\hline $\begin{array}{l}\text { Impedance } \\
\text { poles, } \varphi_{Z}\end{array}$ & $1.0565 \pi / 2$ & $3.021 \pi / 2$ & $5.005 \pi / 2$ & $7 \pi / 2$ & $9 \pi / 2$ \\
$\varphi_{Z} / \varphi_{Y}$ & 1.0565 & 1.0066 & 1.0024 & 1.0012 & $\begin{array}{l}1.000 \\
7\end{array}$ \\
\hline
\end{tabular}

\section{Internal Damping Effects}

Materials under dynamic operation displays internal heating due to several loss mechanisms (Lazan, 1968). Such losses can be incorporated in the mathematical model of the piezoelectric active sensor by assuming complex expressions for the compliance and dielectric constant:

$$
\bar{s}_{11}=s_{11}(1-\mathrm{i} \eta), \quad \bar{\varepsilon}_{33}=\varepsilon_{33}(1-\mathrm{i} \delta)
$$

The values of $\eta$ and $\delta$ vary with the piezoceramic formulation, but are usually small $(\eta, \delta<5 \%)$. The admittance and impedance become complex expressions:

$$
\begin{gathered}
\bar{Y}=\mathrm{i} \omega \cdot \bar{C}\left[1-\bar{\kappa}_{31}^{2}\left(1-\frac{1}{\bar{\varphi} \cot \bar{\varphi}}\right)\right] \\
\bar{Z}=\frac{1}{\mathrm{i} \omega \cdot \bar{C}}\left[1-\bar{\kappa}_{31}^{2}\left(1-\frac{1}{\bar{\varphi} \cot \bar{\varphi}}\right)\right]^{-1}
\end{gathered}
$$

where $\bar{\kappa}_{13}^{2}=d_{31}^{2} /\left(\bar{s}_{11} \bar{\varepsilon}_{33}\right)$ is the complex coupling factor, and $\bar{C}=(1-\mathrm{i} \varepsilon) C, \bar{\varphi}=\varphi \sqrt{1-\mathrm{i} \delta}$. Similar expressions can be derived for the breadth and thickness vibrations, with appropriate use of indices. When studying the frequency spectrum of the complex admittance and impedance, an interesting phenomenon is observed at resonance and antiresonance frequencies. Recall that, in the absence of internal damping, the admittance is purely imaginary and, at resonance, crosses over from $+\infty$ to $-\infty$. The effect of damping is to reduce these extremes to finite, though very large values, such that, while traversing a resonance frequency, the imaginary part of admittance crosses from a very large positive to a very large negative value, and, in this process, its value at resonance is actually zero. At the resonance frequency, the real part of the admittance is the only non-zero term, since the response is only limited by the physical dissipation mechanism. At resonance, the real part of admittance displays a clear peak, which can be used to detect the value of the resonance frequency (This phenomenon is well known in vibration testing, as described by, e.g., Ewins, 1984). A similar behavior is also displayed by the complex impedance.

\section{Longitudinal Vibrations of a Clamped Active Sensor}

Consider fully constrained PZT active sensor as shown in Figure 4. The boundary conditions for this case are expressed as $u_{1}\left(-\frac{1}{2} l\right)=u_{1}\left(\frac{1}{2} l\right)=0$. General solution (5) upon substitution into Equation (3):

$$
\begin{gathered}
\gamma\left(C_{1} \cos \frac{1}{2} \gamma l-C_{2} \sin \frac{1}{2} \gamma l\right)=s_{11}^{E} T_{0}+d_{31} \hat{E}_{3} \\
\gamma\left(C_{1} \cos \frac{1}{2} \gamma l+C_{2} \sin \frac{1}{2} \gamma l\right)=-s_{11}^{E} T_{0}+d_{31} \hat{E}_{3}
\end{gathered}
$$




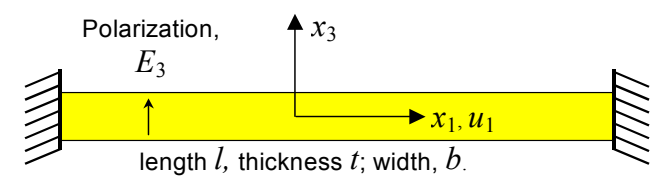

Figure 4 Clamped PZT wafer active sensor.

Upon summation $C_{1}=\frac{d_{31} \hat{E}_{3}}{\gamma \cos \frac{1}{2} \gamma l}$, and the general solution is expressed as:

$$
\hat{u}_{1}(x)=\frac{1}{2} u_{I S A} \frac{\sin \gamma x}{\frac{1}{2} \gamma l \cos \frac{1}{2} \gamma l}
$$

Equation (18) is applied only for the even modes, i.e. for the electromechanical resonances.

Imposing boundary condition into Equation (10), we obtain the electric charge:

$$
\begin{gathered}
Q=\int_{-\frac{l}{2}}^{+\frac{l}{2}} \int_{-\frac{b}{2}}^{+\frac{b}{2}} D_{3} d x d y=\varepsilon_{33}^{T} \frac{b l}{t} V\left[1+\kappa_{31}^{2}\left(\left.\frac{1}{l} \frac{1}{d_{31} E_{3}} u_{1}\right|_{-\frac{l}{2}} ^{+\frac{l}{2}}-1\right)\right], \\
Q=\varepsilon_{33}^{T} C V\left[1-\kappa_{31}^{2}\right]
\end{gathered}
$$

For harmonic motion, $\hat{I}=\mathrm{i} \omega \cdot \hat{Q}$, the electric admittance and impedance expressions for a PZT active constrained sensor are obtained in the following form:

$$
Y=\mathrm{i} \omega \cdot C\left[1-\kappa_{31}^{2}\right], \quad Z=\frac{1}{\mathrm{i} \omega \cdot C}\left[1-\kappa_{31}^{2}\right]^{-1}
$$

It can be shown that this result is equivalent with that mentioned by Ikeda (1996). The effect of structural and sensor damping can be easily introduced in Equations (20) by the use of complex notations:

$$
\ddot{Y}=\mathrm{i} \omega \cdot \bar{C}\left[1-\bar{\kappa}_{31}^{2}\right], \quad Z=\frac{1}{\mathrm{i} \omega \cdot \bar{C}}\left[1-\bar{\kappa}_{31}^{2}\right]^{-1}
$$

\section{Longitudinal Vibrations of an Elastically Constrained Active Sensor}

Consider a constrained PZT active sensor as shown in Figure 5. For symmetry, the overall structural stiffness, $k_{s t r}$, has been split into two end components, each of size $2 k_{s t r}$. The boundary conditions applied at both ends connect the resultant of internal stresses with the spring reaction force, i.e.,

$$
\begin{gathered}
T_{1}\left(\frac{1}{2} l_{a}\right) b_{a} t_{a}=-2 k_{s t r} u_{1}\left(\frac{1}{2} l_{a}\right) \\
T_{1}\left(-\frac{1}{2} l_{a}\right) b_{a} t_{a}=2 k_{s t r} u_{1}\left(-\frac{1}{2} l_{a}\right)
\end{gathered}
$$

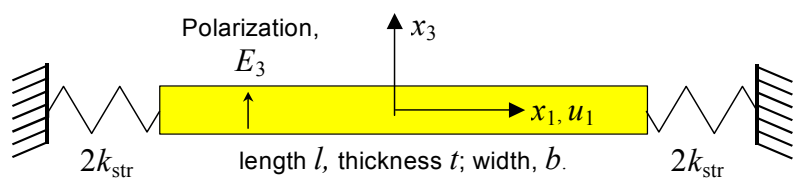

Figure 5 PZT wafer active sensor constrained by an overall structural stiffness, $k_{\text {str. }}$.

Substitution of Equation (22) into Equation (3) gives:

$$
\begin{gathered}
u_{1}^{\prime}\left(\frac{1}{2} l_{a}\right) b_{a} t_{a}=-2 k_{s t r} \frac{s_{11}^{E}}{b t} u_{1}\left(\frac{1}{2} l_{a}\right)+d_{31} E_{3} \\
u_{1}^{\prime}\left(-\frac{1}{2} l_{a}\right) b_{a} t_{a}=2 k_{s t r} \frac{s_{11}^{E}}{b t} u_{1}\left(-\frac{1}{2} l_{a}\right)+d_{31} E_{3}
\end{gathered}
$$

Introducing the quasi-static stiffness of the PZT active sensor,

$$
k_{P Z T}=\frac{A_{a}}{s_{11}^{E} l_{a}}
$$

and the stiffness ratio

$$
r=\frac{k_{s t r}}{k_{P Z T}}
$$

Equation (23) can be re-arranged in the form:

$$
u_{1}^{\prime}\left( \pm \frac{1}{2} l_{a}\right) b_{a} t_{a} \pm \frac{r}{l_{a} / 2} \cdot u_{1}\left(\frac{1}{2} l_{a}\right)=d_{31} E_{3}
$$

Substitution of the general solution (5) yields the following linear system in $C_{1}$ and $C_{2}$ :

$$
\begin{aligned}
& (\varphi \cos \varphi+r \sin \varphi) C_{1}-(\varphi \sin \varphi-r \cos \varphi) C_{2}=\frac{1}{2} u_{I S A} \\
& (\varphi \cos \varphi+r \sin \varphi) C_{1}+(\varphi \sin \varphi-r \cos \varphi) C_{2}=\frac{1}{2} u_{I S A}
\end{aligned}
$$

where $u_{I S A}=d_{31} \hat{E}_{3} \cdot l_{a}$ and $\varphi=\frac{1}{2} \gamma l_{a}$. Upon solution,

$$
\hat{u}_{1}(x)=\frac{1}{2} u_{I S A} \frac{\sin \gamma x}{(\varphi \cos \varphi+r \sin \varphi)}
$$

Substitution into Equation (3) leads to admittance and impedance expressions for a PZT active sensor constrained by the structural substrate with an equivalent stiffness ratio $r$ :

$$
\begin{gathered}
Y=\mathrm{i} \omega \cdot C\left[1-\kappa_{31}^{2}\left(1-\frac{1}{\varphi \cot \varphi+r}\right)\right] \\
Z=\frac{1}{\mathrm{i} \omega \cdot C}\left[1-\kappa_{31}^{2}\left(1-\frac{1}{\varphi \cot \varphi+r}\right)\right]^{-1}
\end{gathered}
$$


Comparison of this equation with the Equations (12) corresponding to the free sensor, indicates that the structural stiffness ratio, $r$, is additive to the sensor resonance term, $\varphi \cot \varphi$. When the PZT active sensor is used in a frequency sweep, the apparent structural stiffness, $k_{s t r}$ will vary with frequency, going through zero at structural resonances, and extreme values at structural anti-resonances. Equations (29) infers that both structural resonances and sensor resonances will be reflected in the admittance and impedance frequency spectra.

Analysis of the asymptotic behavior of Equations (29) reveals important facts. For a vanishingly small $r$, i.e., free sensor vibrations, we get

$$
Y(r) \rightarrow Y_{\text {free }}=\mathrm{i} \omega \cdot C\left[1-\kappa_{31}^{2}\left(1-\frac{1}{\varphi \cot \varphi}\right)\right] \text { as } r \rightarrow 0(30)
$$

For infinitely large $r$, i.e., clamped sensor vibrations, we get Equation (20):

$$
Y(r) \rightarrow Y_{\text {clamped }}=\mathrm{i} \omega \cdot C\left[1-\kappa_{31}^{2}\right] \text { as } r \rightarrow \infty
$$

Both these formulae are consistent with the fundamental theory of piezoelectricity (Ikeda, 1996). On the other hand, as $\gamma_{a} \rightarrow 0$ (i.e., quasi-static sensor conditions), we get

$$
Y_{q s}=\mathrm{i} \omega \cdot C\left[1-\kappa_{31}^{2} \frac{r}{1+r}\right], Z_{q s}=\frac{1}{\mathrm{i} \omega \cdot C}\left[1-\kappa_{31}^{2} \frac{r}{1+r}\right]^{-1},
$$

which is consistent with the results of Liang et al. (1994), Giurgiutiu et al. (1994), and other investigators. The expressions contained in Equations (29) are a generalization of these results and bridge the gap between high-frequency sensor-focused analysis (e.g., Ikeda, 1996) and low-frequency structure-focused analysis (e.g., Liang et al., 1994). The present results cover the complete frequency spectrum and encompass both structure and sensor dynamics.

The effect of structural and sensor damping can be easily introduced in Equations (29) by the use of complex notations:

$$
\begin{aligned}
& \bar{Y}=\mathrm{i} \omega \cdot \bar{C}\left[1-\bar{\kappa}_{31}^{2}\left(1-\frac{1}{\bar{\varphi} \cot \bar{\varphi}+\bar{r}}\right)\right] \\
& \bar{Z}=\frac{1}{\mathrm{i} \omega \cdot \bar{C}}\left[1-\bar{\kappa}_{31}^{2}\left(1-\frac{1}{\bar{\varphi} \cot \bar{\varphi}+\bar{r}}\right)\right]^{-1}
\end{aligned}
$$

where $\bar{r}$ is the frequency-dependent complex stiffness ratio that reflects the structural point-wise dynamics and the sensor dissipation mechanisms.

\section{Breadth and Thickness Vibrations}

The breadth and thickness vibrations could be deduced in ways similar with those used for length vibrations. However, certain aspect-ratio particularities and planestress vs. plane-strain issues need to be addressed (Pugachev et al., 1984; Ikeda, 1996). For a free sensor:

$$
Y_{l}=\mathrm{i} \omega \cdot C\left[1+\kappa_{31}^{2}\left(\frac{\tan \varphi_{l}}{\varphi_{l}}-1\right)\right], Z_{l}=Y_{l}^{-1} \text { (length)(34) }
$$

where $\kappa_{31}^{2}=\frac{d_{31}^{2}}{\varepsilon_{33}^{T} s_{11}^{E}}, \mathrm{v}_{l}=1 / \sqrt{\rho s_{11}^{E}}, \varphi_{l}=\frac{1}{2} \frac{\omega l}{\mathrm{v}_{l}}$, .

$$
Y_{b}=\mathrm{i} \omega \cdot C\left[1+\kappa_{31}^{\prime 2}\left(\frac{\tan \varphi_{b}}{\phi_{b}}-1\right)\right], \quad Z_{b}=Y_{b}^{-1} \quad \text { (breadth) }
$$

where $\quad \kappa_{31}^{\prime 2}=\frac{\kappa_{31}^{2}}{1-\kappa_{31}^{2}} \frac{1+\sigma}{1-\sigma}, \quad \mathrm{v}_{b}=1 / \sqrt{\rho s_{11}^{2}\left(1-\sigma^{2}\right)}$, $\varphi_{b}=\frac{1}{2} \frac{\omega b}{\mathrm{v}_{b}},, \sigma=$ Poisson's ratio.

$$
Z_{t}=\frac{1}{\mathrm{i} \omega} \frac{t}{b l \varepsilon_{33}^{S}}\left[1-\kappa_{t}^{2} \frac{\tan \varphi_{t}}{\varphi_{t}}\right], Y_{t}=Z_{t}^{-1} \text { (thickness)(36) }
$$

where $\kappa_{t}^{2}=\frac{h_{33}^{2}}{\beta_{33}^{S} c_{33}^{D}}, \mathrm{v}_{t}=\sqrt{c_{33}^{D} / \rho}, \varphi_{t}=\frac{1}{2} \frac{\omega t}{\mathrm{v}_{t}}$.

\section{Axisymmetric Vibrations}

PZT wafer active sensors are also produced in circular shape. To cater for this situation, the axisymmetric radial vibrations of a thin piezoelectric disk (Figure 6) are considered. Because of axial symmetry, this twodimensional motion can be treated with a one-dimensional model in which the displacement varies with radius only. The solution of the corresponding differential equation is expressed in terms of Bessel functions (IEEE Std. 176 1987). the displacement $\xi(r)$ at radial position $r$ is given by

$$
\xi(r)=A J_{1}\left(\omega r / v_{p}\right) \cdot e^{j \omega t}
$$

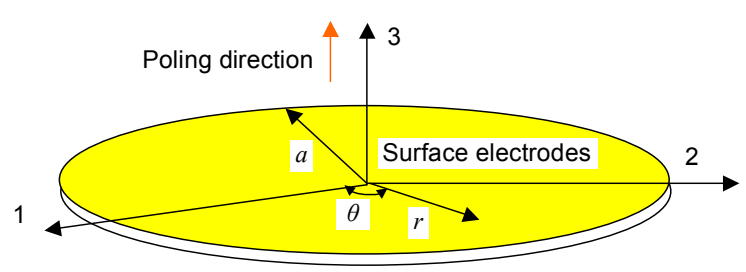

Figure 6 Schematic of a PZT disk wafer 
where $J_{1}$ is the first order Bessel function while $v_{p}=1 / \sqrt{\rho s_{11}^{E} \cdot\left(1-\sigma^{2}\right)}$ is the wave speed for axisymmetric radial motion. Upon solution for free-edge boundary conditions, one can express the electrical admittance (Ikeda, 1996) as

$$
Y_{d}=j \omega C \cdot \frac{k_{p}^{2}}{1-k_{p}^{2}} \cdot \frac{(1+\sigma)}{\varphi_{d} J_{0}(\varphi) / J_{1}\left(\varphi_{d}\right)-(1-\sigma)}
$$

where $\varphi_{d}=\omega a / v_{p}, a$ is the radius of a disk, and $k_{p}=\sqrt{2 d_{31}^{2} /\left[s_{11}^{E} \cdot(1-\sigma) \varepsilon_{33}^{T}\right]}$ is the planar coupling factor.

\section{MODELING OF A PZT ACTIVE SENSOR INSTALLED ON A STRUCTURAL SUBSTRATE}

After reviewing the dynamics of the sensor alone under various boundary conditions, we now turn our attention to the dynamics of a PZT active sensor installed on a structure. The dynamics of the structural substrate and its interaction with the sensor dynamics is paramount in this analysis.

To couple the sensor and the structure, we are going to use Equations (25) and (29) where the dynamic structural stiffness, $k_{s t r}(\omega)$, will be used in Equation (25). To derive $k_{s t r}(\omega)$, we calculate the response of structural substrate using the general theory of structural vibrations (Timoshenko, 1955; Meirovitch, 1986; Inman, 1996; and Kelly, 2000) However, the PZT excitation departs from the typical textbook formulation since it acts a pair of selfequilibrating axial forces and bending moments that are separated by a small finite distance, $l_{P Z T}$, as shown next.

\section{Definition of the Excitation Forces and Moments}

The excitation forces and moments acting upon the beam structure are derived from the PZT force, $F_{P Z T}=\hat{F}_{P Z T} e^{i \omega t}$, using the beam cross-section geometry (Figure 7):

$$
M_{a}=F_{P Z T} \frac{h}{2}, \quad N_{a}=F_{P Z T}
$$

The space-wise distribution of excitation bending moment and axial force are expressed using the Heaviside function, $H\left(x-x_{a}\right)$, defined as $H\left(x-x_{a}\right)=0$ for $x<x_{a}$, and $H\left(x-x_{a}\right)$ $=1$ for $x_{a} \leq x$ :

$$
\begin{gathered}
N_{e}(x, t)=N_{a}\left[-H\left(x-x_{a}\right)+H\left(x-x_{a}-l_{a}\right)\right] \cdot e^{i \omega t} \\
M_{e}(x, t)=-M_{a}\left[-H\left(x-x_{a}\right)+H\left(x-x_{a}-l_{a}\right)\right] \cdot e^{i \omega t}
\end{gathered}
$$

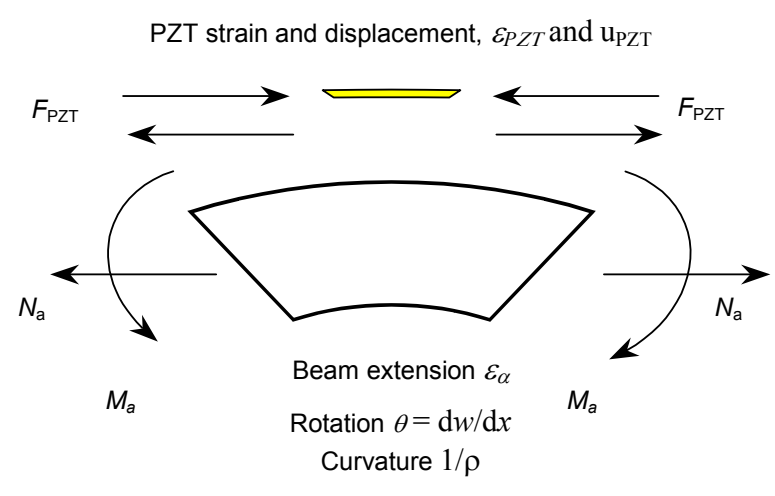

Figure 7 Interaction between PZT active sensor and a substructure: forces and moments.

where subscript $e$ signifies excitation. Equations (40) and (41) correspond to axial and flexural vibrations, respectively. Axial vibrations modes are usually of much larger frequency than flexural vibration modes, and were neglected by previous researchers (Liang et al., 1994). However, their vibration frequencies are commensurable with those of the PZT active sensors. Other researchers have only considered axial modes and neglected the flexural vibrations (Park, Cudney and Inman, 2000).

\section{Calculation of Frequency Response Function and Dynamic Structural Stiffness}

To obtain the dynamic structural stiffness, $k_{s t r}$, presented by the structure to the PZT, we first calculate the elongation between the two points, $\mathrm{A}$ and $\mathrm{B}$, connected to the PZT ends. Simple kinematics gives the horizontal displacement of a generic point $P$ place on the surface of the beam:

$$
u_{P}(t)=u(x)-\frac{h}{2} w^{\prime}(x),
$$

where $u$ and $w$ are the axial and bending displacements of the neutral axis. Letting $\mathrm{P}$ be $\mathrm{A}$ and $\mathrm{B}$, and taking the difference, yields:

$$
\begin{aligned}
& u_{P Z T}(t)=u_{B}(t)-u_{A}(t)= \\
& =u\left(x_{a}, t\right)-u\left(x_{a}+l_{a}, t\right)-\frac{h}{2}\left[w^{\prime}\left(x_{a}, t\right)-w^{\prime}\left(x_{a}+l_{a}, t\right)\right]
\end{aligned}
$$

Using classical axial and flexural vibrations analysis, one writes $\quad u(x, t)=e^{\mathrm{i} \omega t} \sum_{n_{u}} \frac{C_{n_{u}}}{\omega_{n_{u}}^{2}-\omega^{2}+\mathrm{i} \cdot 2 \zeta_{n_{u}} \omega} U_{n_{u}}(x) \quad$ and $w^{\prime}(x, t)=e^{\mathrm{i} \omega t} \sum_{n_{w}} \frac{C_{n_{w}}}{\omega_{n_{w}}^{2}-\omega^{2}+\mathrm{i} \cdot 2 \zeta_{n_{w}} \omega} W_{n_{w}}^{\prime}(x)$, where $U_{n_{u}}(x)$ are the axial modes and $W_{n_{w}}(x)$ are the bending modes. Equations (40) and (41) can be used to express the 
constants $C_{n_{u}}$ and $C_{n_{W}}$ in terms of the excitation forces and moments (Giurgiutiu and Zagrai, 2001). Hence, Equation (43) becomes

$$
\hat{u}_{P Z T}=\frac{\hat{F}_{P Z T}}{\rho A}\left\{\begin{array}{l}
\sum_{n_{u}} \frac{\left[U_{n_{u}}\left(x_{a}+l_{a}\right)-U_{n_{u}}\left(x_{a}\right)\right]^{2}}{\omega_{n_{u}}^{2}-\omega^{2}+\mathrm{i} \cdot 2 \zeta_{n_{u}} \omega_{n_{u}} \omega}+ \\
+\left(\frac{h}{2}\right)^{2} \sum_{n_{w}} \frac{\left[W_{n_{w}}^{\prime}\left(x_{a}+l_{a}\right)-W_{n_{w}}^{\prime}\left(x_{a}\right)\right]^{2}}{\omega_{n_{w}}^{2}-\omega^{2}+\mathrm{i} \cdot 2 \zeta_{\mathrm{n}_{w}} \omega_{n_{w}} \omega}
\end{array}\right\}
$$

where $\hat{u}_{P Z T}$ is the vibration amplitude, i.e., $u_{P Z T}(t)=\hat{u}_{P Z T} e^{i \omega t}$. Dividing Equation (44) by $\hat{F}_{P Z T}$ yields the structural frequency response function (FRF) to the Single Input Single Output (SISO) excitation applied by the PZT active sensor. This situation is similar to conventional modal testing (Harris, 1996, Section 21) with the proviso that the PZT wafers are unobtrusive and permanently attached to the structure. The dynamic structural stiffness is the inverse of frequency response function, i.e.,

$$
k_{s t r}(\omega)=\frac{\hat{F}_{P Z T}}{\hat{u}_{P Z T}}=\rho A\left\{\begin{array}{l}
\sum_{n_{u}} \frac{\left[U_{n_{u}}\left(x_{a}+l_{a}\right)-U_{n_{u}}\left(x_{a}\right)\right]^{2}}{\omega_{n_{u}}^{2}-\omega^{2}+\mathrm{i} \cdot 2 \zeta_{n_{u}} \omega_{n_{u}} \omega}+ \\
+\left(\frac{h}{2}\right)^{2} \sum_{n_{w}}^{2} \frac{\left[W_{n_{w}}^{\prime}\left(x_{a}+l_{a}\right)-W_{n_{w}}^{\prime}\left(x_{a}\right)\right]^{2}}{\omega_{n_{w}}^{2}-\omega^{2}+\mathrm{i} \cdot 2 \zeta_{n_{w}} \omega_{n_{w}} \omega}
\end{array}\right\}^{-1}
$$

For free-free beams (Inman, 1996) axial and flexural components are:

$$
\begin{aligned}
& U_{n_{u}}(x)=A_{n_{u}} \cos \left(\gamma_{n_{u}} x\right), \quad A_{n_{u}}=\sqrt{2 / l}, \quad \gamma_{n_{u}}=\frac{n_{u} \pi}{l}, \\
& \omega_{n_{u}}=\gamma_{n_{u}} c, c=\sqrt{\frac{E}{\rho}}, n_{u}=1,2, \ldots \\
& W_{n_{w}}(x)=A_{n_{w}}\left[\cosh \gamma_{n_{W}} x+\cos \gamma_{n_{w}} x-\sigma_{n_{w}}\left(\sinh \gamma_{n_{w}} x+\sin \gamma_{n_{w}} x\right)\right] \\
& \omega_{n_{w}}=\gamma_{n_{w}}^{2} a, \quad \sqrt{-}, A_{n_{w}}=1 / \sqrt{\int_{0}^{l} W_{n_{w}}^{2}(x) d x}
\end{aligned}
$$

Numerical values of $l \cdot \gamma_{n_{w}}$ and $\sigma_{n_{w}}$ for $n_{w} \leq 5$ can be found in Blevins (1979), page 108; for $5<n_{w}$, $\gamma_{n_{w}}=\frac{1}{l} \frac{(2 n+1) \pi}{2}$ and $\sigma_{n_{w}}=1$.

The derivations (29), (45), (46), (47) open the way for the numerical example and comparison between theoretical and experimental results.

\section{NUMERICAL SIMULATION OF PZT ACTIVE SENSOR RESPONSE}

To illustrate our analysis, we performed numerical simulation of a typical piezoelectric active sensor with following dimensions: $l=6.99 \mathrm{~mm}, b=1.65 \mathrm{~mm}, t=0.2$ $\mathrm{mm}$. The piezoceramic properties are presented in Table 2 (Note that $\varepsilon_{0}=8.84194 \mathrm{pF} / \mathrm{m}$ ).

Figure 8 presents the numerical simulation of longitudinal vibrations over a wide frequency range. The electromechanical admittance and impedance, as it would be measured at sensor terminals, are plotted. In these simulations, $1 \%$ damping ( $\delta=\varepsilon=1 \%$ ) was assumed. The introduction of this slight damping values generated nonsingular behavior around the resonance and anti-resonance points and gave realism to our simulation. The examination of Figure 8 shows the effect of resonances and antiresonances on the admittance and impedance curves. Outside resonances, the electro-mechanical admittance behaves essentially like i $\omega C$ (Figure 8a). Similarly, outside anti-resonances, the electro-mechanical impedance behaves like $1 /(\mathrm{i} \omega C)$. At resonances and anti-resonances, the basic $\mathrm{i} \omega C \mathrm{C} /(\mathrm{i} \omega C)$ patterns of behavior are modulated by the resonant and anti-resonant responses that generate zigzags in the imaginary parts and sharp peaks in the real part. The resonance and anti-resonance frequencies can be clearly identified as definite peaks in the real-part plots of the admittance and impedance functions (Figure $8 \mathrm{~b}$ ). These frequencies, read to 4-digit accuracy, are listed in Table 3. Also listed in Table 3 are the undamped resonance and anti-resonance frequencies determined with the $\varphi_{Y}$ and $\varphi_{Z}$ values of Table 1 . Comparison of slightly damped and undamped frequencies reveals that their numerical values remain virtually unchanged when slight damping was introduced. This confirms that the peaks of the admittance and impedance real-part spectra can be confidently used to determine the resonance and anti-resonance frequencies. This has important experimental significance, since extracting the same information from the imaginary part plots is much less practical because, in these plots, the resonance and anti-resonance specific patterns are masked by the dominant $\mathrm{i} \omega C$ and $1 /(\mathrm{i} \omega C)$ basic response. 
Table 2 Properties of piezoelectric ceramic APC-850 (as from the company website www.americanpiezo.com )

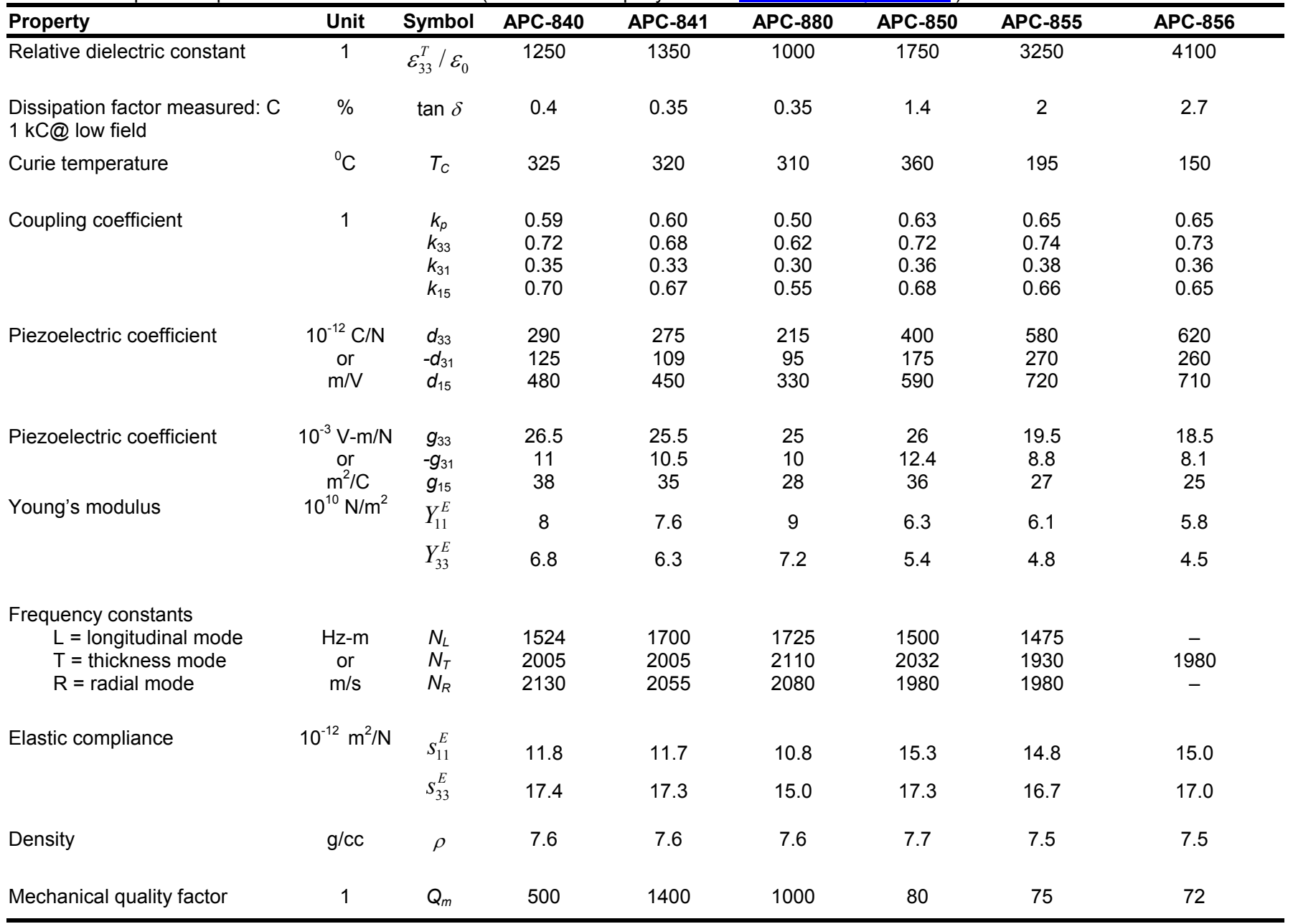

Table 3 Peaks of the admittance and impedance real-part plots; numerical simulation of a PZT active sensor with $I=7 \mathrm{~mm}, b=1.68 \mathrm{~mm}$, $t=0.2 \mathrm{~mm}$, APC-850 piezoceramic, $\delta=\varepsilon=1 \%$.

\begin{tabular}{|c|c|c|c|c|c|}
\hline Mode No. & 1 & 2 & 3 & 4 & 5 \\
\hline Slightly damped resonance frequencies, $f_{Y}, \mathrm{kHz}$. & 208.059 & 624.17 & 1040.29 & 1456.41 & 1872.54 \\
\hline Undamped resonance frequencies, $f_{Y^{*}}, \mathrm{kHz}$. & 208.056 & 624.16 & 1040.28 & 1456.39 & 1872.51 \\
\hline Error in $f_{Y}$ vs. $f_{Y^{*}}, \%$ & $0.0014 \%$ & $0.0016 \%$ & $0.0010 \%$ & $0.0014 \%$ & $0.0016 \%$ \\
\hline Slightly damped anti-resonance frequencies, $f_{z}, \mathrm{kHz}$ & 219.89 & 628.35 & 1042.84 & 1458.26 & 1873.99 \\
\hline Undamped anti-resonance frequencies, $f_{Z^{*}}, \mathrm{kHz}$ & 219.88 & 628.32 & 1042.78 & 1458.18 & 1873.90 \\
\hline Error in $f_{Z}$ vs. $f_{Z^{*}}, \%$ & $0.0045 \%$ & $0.0048 \%$ & $0.0058 \%$ & $0.0055 \%$ & $0.0048 \%$ \\
\hline
\end{tabular}



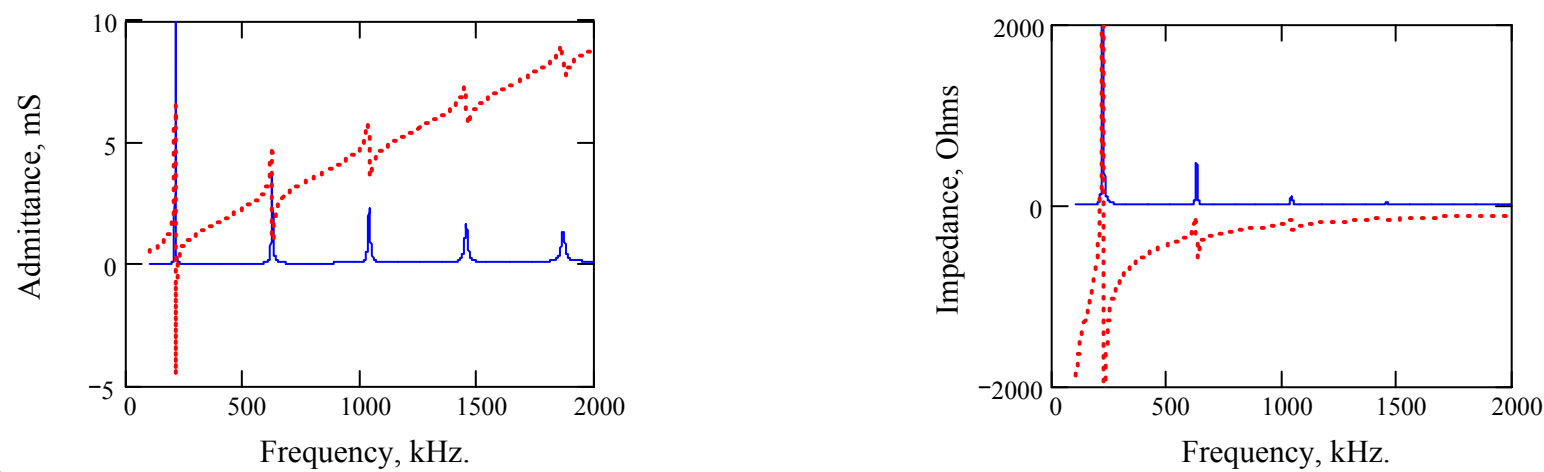

(a)
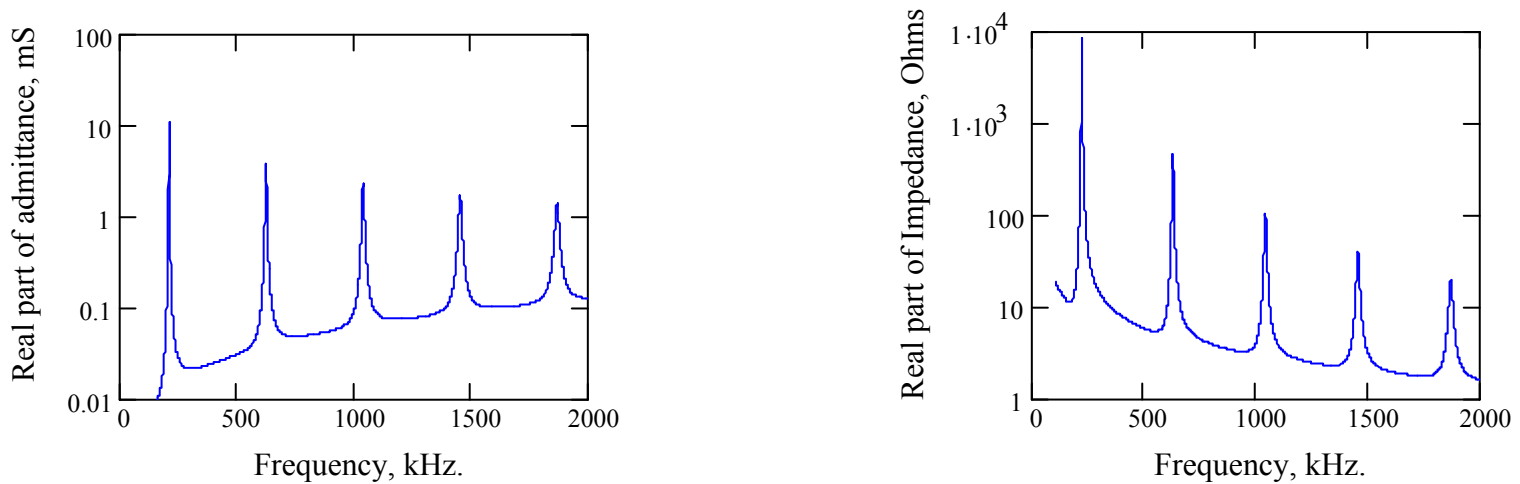

Figure 8 Simulated electromechanical admittance and impedance of a PZT active sensor $\left(\mathrm{l}=7 \mathrm{~mm}, \mathrm{~b}=1.68 \mathrm{~mm}, \mathrm{t}=0.2 \mathrm{~mm}, \varepsilon_{33}^{T}=15.470 \cdot 10^{9} \mathrm{~F} / \mathrm{m}\right.$, $s_{11}^{E}=15.3 \cdot 10^{-12} \cdot \mathrm{Pa}^{-1}, d_{31}=-175 \cdot 10^{-12} \cdot \mathrm{m} / \mathrm{N}, \mathrm{k}_{31}=0.36 \delta=\varepsilon=1 \%$ ): (a) complete plots showing both real (full line) and imaginary (dashed line) parts; (b) plots of real part only, log scale.

\section{PZT ACTIVE SENSORS FABRICATION, AND CHARACTERIZATION}

Previous efforts (Giurgiutiu and Zagrai, 2000) have shown that consistent sensor fabrication and installation procedures are needed for successful implementation of active sensor techniques. Hence, the analysis of sensor fabrication, and the identification/elimination of fabrication faults and shortcomings was one of our major concerns during the present investigation. A batch of 25 APC- 850 small PZT wafers (7 mm sq., $0.2 \mathrm{~mm}$ thick, silver electrodes on both sides), from American Piezo Ceramics, was acquired, and subjected to statistical evaluation. Our selection of these products was based on their affordable cost and adequate manufacturing tolerances (Table 4). When connected to lead wires and adhesively installed on the structural specimen, these wafers would become piezoelectric active sensors. The aim of our investigation was to establish an in-process test procedure that would allow the final properties of the installed active sensor to be traced back to the initial properties of the PZT wafer, as modified by the sensor fabrication process. In this process, we started with the mechanical and electrical properties declared by the vendor, and then conducted our own measurements to verify the vendor data and evaluate its veridicality. The material properties of the basic PZT material are given in Table 2. The mechanical tolerances of these wafers, as presented by the vendor on their website, are given in Table 4. Other tolerances declared by the vendor were $\pm 5 \%$ for resonance frequency, $\pm 20 \%$ for capacitance and $\pm 20 \%$ for the $d_{33}$ constant. For in-process quality assurance of active sensor fabrication, we selected the following indicators: (a) geometrical dimensions; (b) electrical capacitance; (c) E/M impedance and admittance spectra. The geometric measurements were the initial indicators that, when showing an acceptable tolerance and a narrow spread, would build up the investigator's confidence. The electrical capacitance was use to verify electrical consistency of the fabrication process. It was found to be an important but also elusive indicator. We found that during the adhesive installation of the sensor onto the structural specimen, the electrical capacitance will typically decrease, but remain within the order of magnitude of the initial reading. Of the three indicators, the $\mathrm{E} / \mathrm{M}$ impedance and admittance spectra were found to be the most labor-intensive but also the most comprehensive. 
Table 4 Manufacturing tolerances for APC, Inc. small piezoelectric wafers (www.americanpiezo.com )

\begin{tabular}{lcc}
\hline \multicolumn{1}{c}{ Dimension } & Units $(\mathrm{mm})$ & $\begin{array}{c}\text { Standard } \\
\text { Tolerance }\end{array}$ \\
\hline Length or breadth of plates & $<13 \mathrm{~mm}$ & $+/-0.13 \mathrm{~mm}$ \\
Thickness of all parts & $0.20 \mathrm{~mm}$ to & $+/-0.025 \mathrm{~mm}$ \\
\hline
\end{tabular}

\section{Geometric measurements}

Twenty-five nominally identical APC-850 wafers were measured with precision instrumentation consisting of Mitutoyo Corp. CD - 6" CS digital caliper (0.01-mm precision) and Mitutoyo Corp. MCD - 1" CE digital micrometer (0.001-mm precision). Length, breadth, and thickness were measured and recorded. Statistical analysis of the data obtained from these measurements shown good agreement with the Normal distribution (Gauss law). Mean and standard deviation values for length/breadth and thickness were $6.9478 \mathrm{~mm}, \pm 0.5 \%$, and $0.2239 \mathrm{~mm}, \pm 1.4$ $\%$, respectively.

\section{Electrical Measurements}

Electrical capacitance was measured with a BK Precision ${ }^{\circledR}$ Tool Kit ${ }^{\mathrm{TM}} 27040$ digital multimeter with a resolution of $1 \mathrm{pF}$. Capacitance measurements were selected as a in-process quality check to be applied during each step of sensor development and also during the sensor installation process. Capacitance of the basic PZT sensors was measured directly by putting the PZT square on a flat metallic ground plate. The negative probe was connected to the plate in a semi-permanent fashion (hole, bolt + nut + washer, short multifilament lead, alligator clip), and the top of the PZT wafer was touched with the positive probe. Then, data was taken when the tester readings had converged to a stable value. At least 6 readings were recorded and the average was taken. The process was iteratively improved until consistent results were obtained. The results of statistical analysis for direct capacitance test is presented on Figure 8. Mean and standard deviation values are $3.276 \mathrm{nF}, \pm 3.8 \%$.

\section{INTRINSIC E/M IMPEDANCE AND ADMITTANCE CHARACTERISTICS OF THE PZT ACTIVE SENSOR}

The intrinsic E/M impedance/admittance of the PZT active sensor is an important dynamic descriptor for characterizing the sensor prior to its installation on the structure. In our work, the intrinsic $\mathrm{E} / \mathrm{M}$ impedance/admittance of the PZT active sensor was determined both theoretically and experimentally. Theoretical calculations were performed with Equations (34)-(36) The measurements were done with HP 4194A Impedance Phase Gain Analyzer.

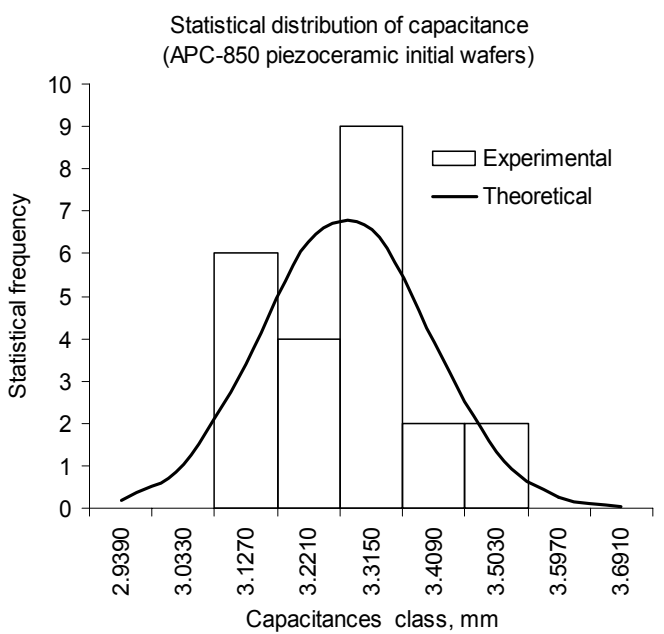

Figure 9 Statistical distribution of APC-850 piezoceramic wafers capacitance.

(a)
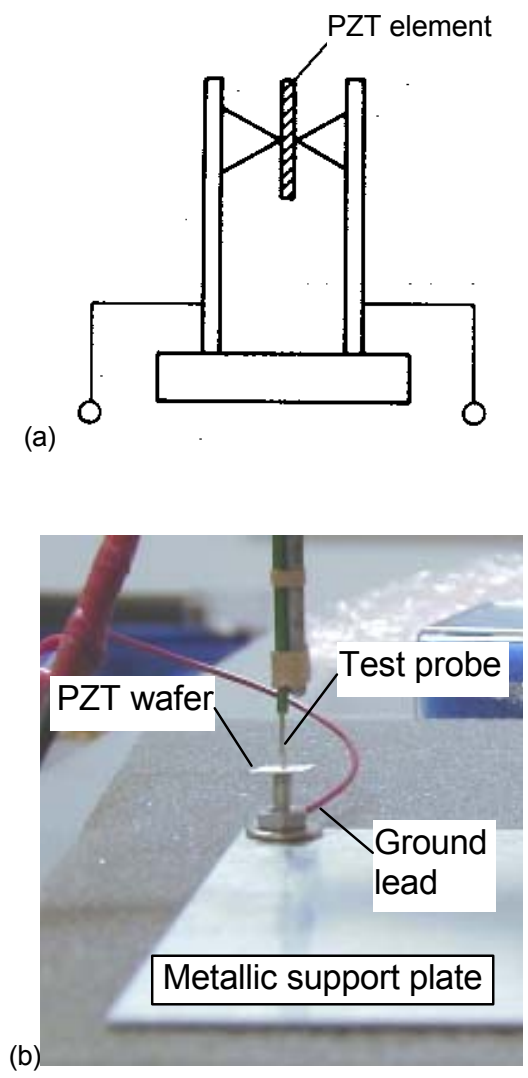

Figure 10 (a) Test jig schematics for dynamic measurement of PZT elements that ensures unrestraint support of the PZT wafer (Waanders, 1991); (b) physical implementation of the schematic as used in our experiments. 
(a)
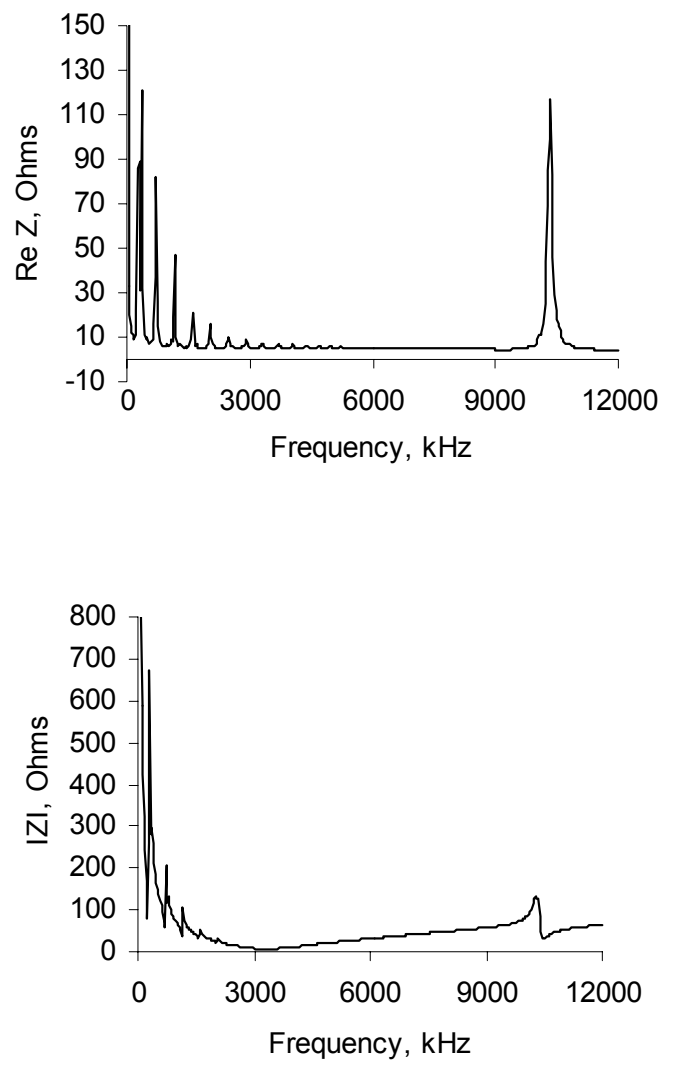

(c)

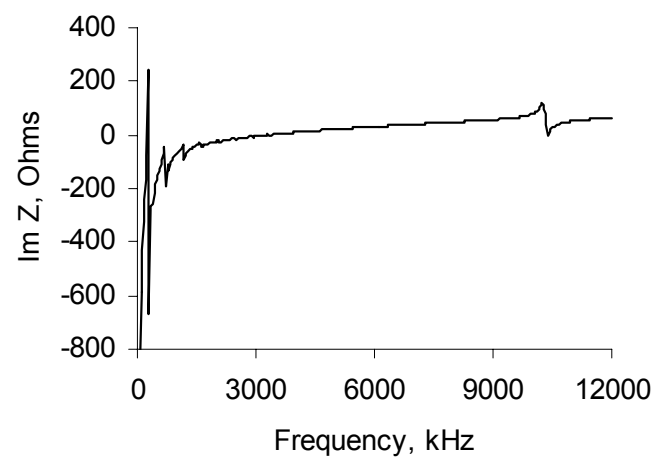

(b)

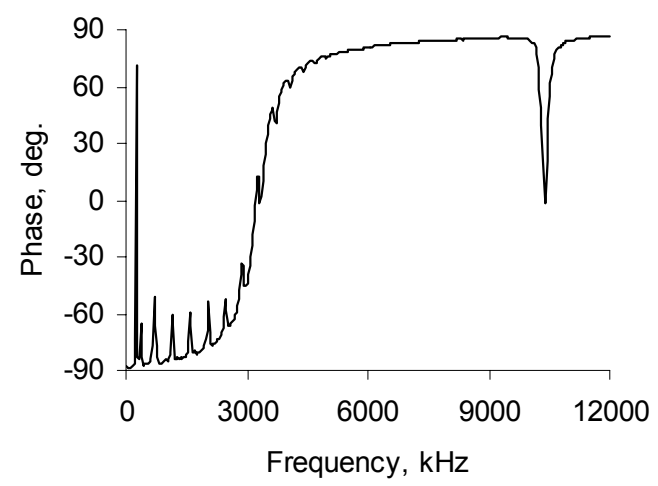

(d)

Figure11 Intrinsic E/M impedance characteristics of the PZT active sensor: (a) real part; (b) imaginary part; (c) amplitude; (d) phase.
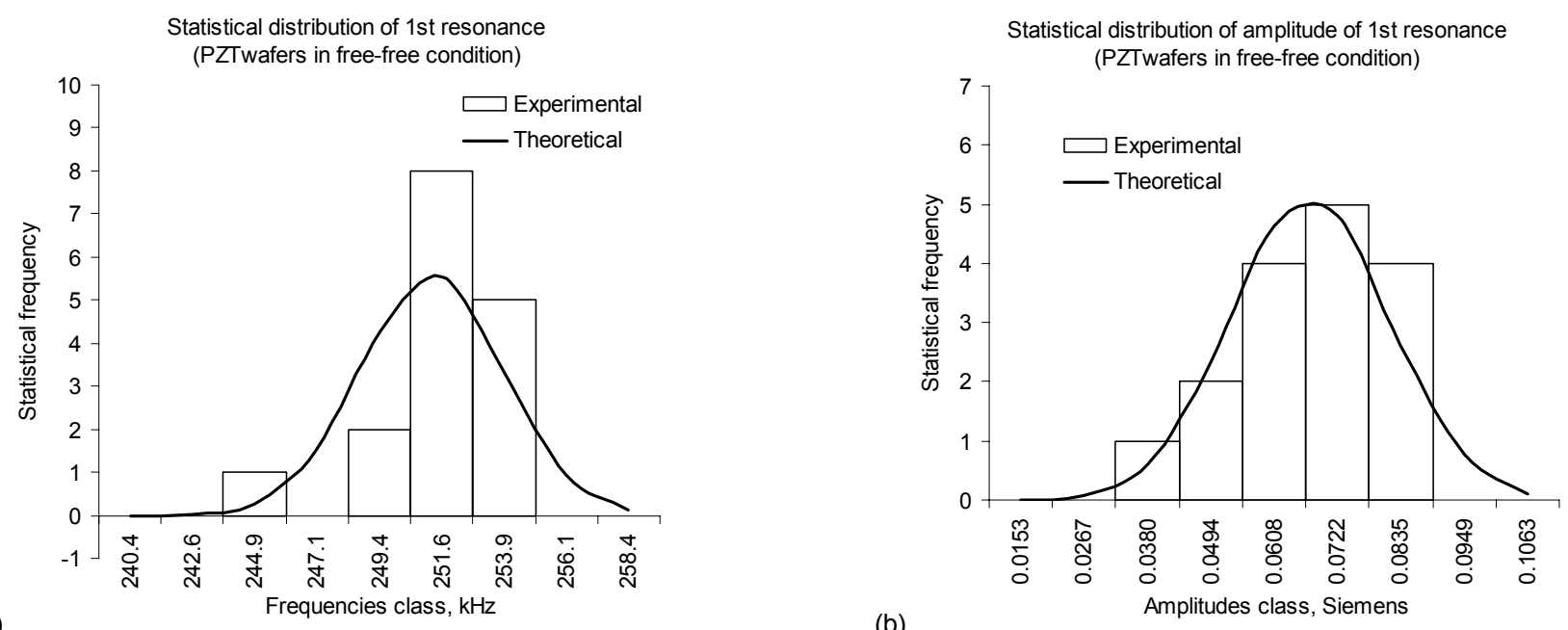

(a)

(b)

Figure 12 Results histograms vs. frequency and amplitude sorting classes: (a) $1^{\text {st }}$ resonance frequencies and (b) admittance amplitudes at $1^{\text {st }}$ resonance of PZT active sensor. 


\section{Measurements of the intrinsic $\mathrm{E} / \mathrm{M}$ impedance and admittance characteristics of the PZT active sensor}

The test fixture for measuring the intrinsic $\mathrm{E} / \mathrm{M}$ impedance/admittance of free PZT active sensor was designed following Waanders (1991) concept shown on Figure 10a. We used a metallic plate with a lead connected at one corner (Figure 10b). The PZT wafer was centered on the bolt head and held in place with the probe tip. Thus, proper support conditions were simulated, and the PZT wafer could freely perform its vibrations. The PZT active sensors were tested in the $100 \mathrm{~Hz}-12 \mathrm{MHz}$ frequency range using the HP 4194A Impedance Analyzer. Typical admittance and impedance frequency spectra are given in Figure 11.

The PZT wafer resonance frequencies were identified from the $\mathrm{E} / \mathrm{M}$ admittance spectra. It was found that since the length and the breadth of the PZT wafers are nearly identical, the corresponding lengthwise and breadthwise frequencies are coalescent, forming twin-peaks of in-plane vibration resonances. The first, second, and third in-plane resonance frequencies, as well as the out-of-plane (thickness) resonance (which is at much higher frequency) were identified and recorded. Also recorded were the values of the corresponding resonance peaks. Statistical distributions of the resonance frequencies and resonance amplitudes are given in Figure 12. Mean values of $251 \mathrm{kHz}$ and $67.152 \mathrm{mS}$, and standard deviations of $\pm 1.2 \%$ and $\pm 21 \%$, respectively, were obtained. These results proved that the basic APC-850 piezoelectric wafers had acceptable quality with a narrow dispersion band in resonance frequency.

\section{Comparison of Measured and Calculated E/M admittance Spectra of PZT Active Sensors}

Measured results and calculated predictions for circular disc and rectangular plate PZT wafer active sensors of various aspect ratios are given in Table 5. Superposed plots of the measured and calculated $\mathrm{E} / \mathrm{M}$ admittance spectra are given in Figures 13 through 16. Figure 13 refers to a circular disk active sensor, while Figures 14 - 16 refer to rectangular active sensors of various aspect ratios.

\section{E/M Admittance Spectrum for a Circular Disk Active Sensor}

The E/M admittance of a disk-shaped piezoelectric active sensor undergoing axisymmetric in-plane radial vibrations was modeled with Equation (38). Figure 13 shows superposed the predicted and measured results. On this plot, three resonance peaks are clearly visible. The corresponding frequencies $(300 \mathrm{kHz}, 784 \mathrm{kHz}$, and $1,247 \mathrm{kHz}$, as indicated in Table 5) correspond to the first three in-plane radial modes. The forth in-plane frequency $(1,697 \mathrm{kHz})$ which lies outside the $0-1,500 \mathrm{kHz}$ plotting range, was not plotted. However, its value appears in Table 5. During our experiments, we also noticed a very high frequency peak in the E/M impedance real-part response at 10,895 kHz (Figure 12a). This value can be identified with the out-of-plane thickness vibration, as predicted by Equation (36). Comparison of measured and calculated results listed in Table 5 for the circular disk case indicate very good agreement between theory and experiments $(2.1 \%$ maximum error).

\section{E/M Admittance Spectrum for Rectangular Plate Active Sensors}

As received, the rectangular piezoceramic wafers had an aspect ratio close to one, i.e., were practically square. The modeling of in-plane vibrations of square PZT wafers is not easily attainable, since no closed-form solution exists for this 2-dimensional (2-D) nonaxisymmetric mode. In our analysis, we investigated the possibility of using 1-dimensional (1-D) results of Equations (34-36) to predict the E/M impedance and admittance response, and subsequently identify resonance frequencies. To achieve this, we progressively used active sensor specimens of increasing aspect ratios $1: 1,2: 1$, and $4: 1$. The specimens were fabricated from an as-received square wafer, by machining the breadth in half and then in quarter. It was expected that, as the aspect ratio increases, the experimental results would converge with 1-dimensional prediction

Figure 14 shows the admittance real-part spectrum for the square-shaped piezoelectric active sensor (aspect ratio $1: 1)$. In the $0-1500 \mathrm{kHz}$ frequency band, the 1-D theoretical curve predicts 3 double peaks $(1 \mathrm{~L}, 1 \mathrm{~B}, 2 \mathrm{~L}$, $2 \mathrm{~B}, 3 \mathrm{~L}, 3 \mathrm{~B})$ and one single peak $(4 \mathrm{~L})$ where $\mathrm{L}=$ length modes, $\mathrm{B}=$ breadth modes. The $4 \mathrm{~B}$ mode is outside the $0-1500 \mathrm{kHz}$ range, and hence was not plotted. The double peaks indicate the modes coalescence between length and breadth vibrations due to $l=7.00 \mathrm{~mm}$ and $b=$ $6.99 \mathrm{~mm}$ giving almost identical length and breadth frequencies at the low end of the spectrum. The corresponding resonance frequencies (Table 5) are 208 $\mathrm{kHz}(1 \mathrm{~L}), 222 \mathrm{kHz}(1 \mathrm{~B}), 621 \mathrm{kHz}(2 \mathrm{~L}), 663 \mathrm{kHz}(2 \mathrm{~B})$, $1038 \mathrm{kHz}(3 \mathrm{~L}), 1108 \mathrm{kHz}(3 \mathrm{~B})$ and $1451 \mathrm{kHz}(4 \mathrm{~L})$. It should be noted that the calculated frequencies are in the harmonic ratio 1:3:5:7, as predicted by Table 3 . The $1 \mathrm{~L}$ and $1 \mathrm{~B}$ experimental results are significantly different from the theoretical predictions. The high positive errors (19\% and 37\%, respectively) are indicative of the 2-D stiffening effect, typical of low-aspect ratio in-plane vibrations, that is not captured by the 1-D theory. At higher modes, this stiffening diminishes, and the agreement between theory and experiment improves (7.3\% and $5.6 \%$ for the $2 \mathrm{~L}$ and $2 \mathrm{~B}$ modes; $2.3 \%$ and $3.6 \%$ for the $3 \mathrm{~L}$ and $3 \mathrm{~B}$ modes). This indicates that, with the exception of first modes, 1-D theory gives a reasonable approximation at aspect ratios as low as 1:1. Also in good agreement is the out-of-plane 1T thickness frequency $(10,565 \mathrm{kHz}, 0.7 \%$ error). 


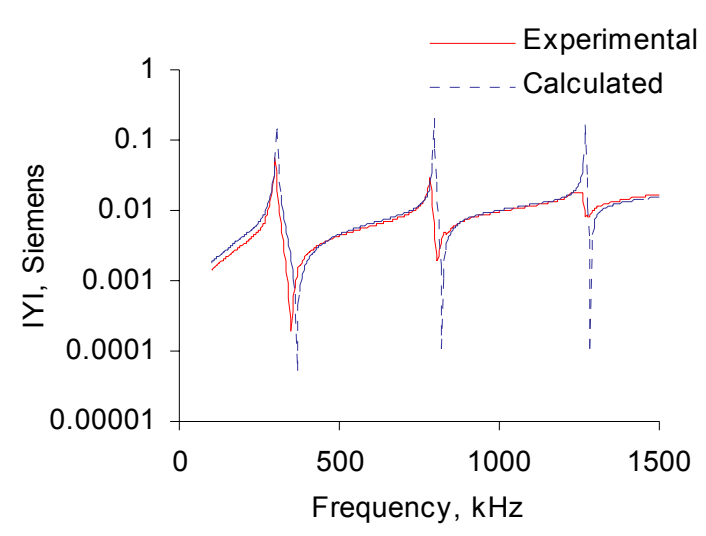

Figure 13 Experimental and calculated admittance spectra for circular wafer active sensor $(\mathrm{d}=6.98 \mathrm{~mm}, \mathrm{t}=$ $0.216 \mathrm{~mm}, \quad \varepsilon_{33}^{T}=15.470 \cdot 10^{9} \mathrm{~F} / \mathrm{m}, \quad s_{11}^{E}=18 \cdot 10^{-12} \cdot \mathrm{Pa}^{-1}, d_{31}=$. $\left.175 \cdot 10^{-12} \cdot \mathrm{m} / \mathrm{V}, \mathrm{k}_{\mathrm{p}}=0.63\right)$.

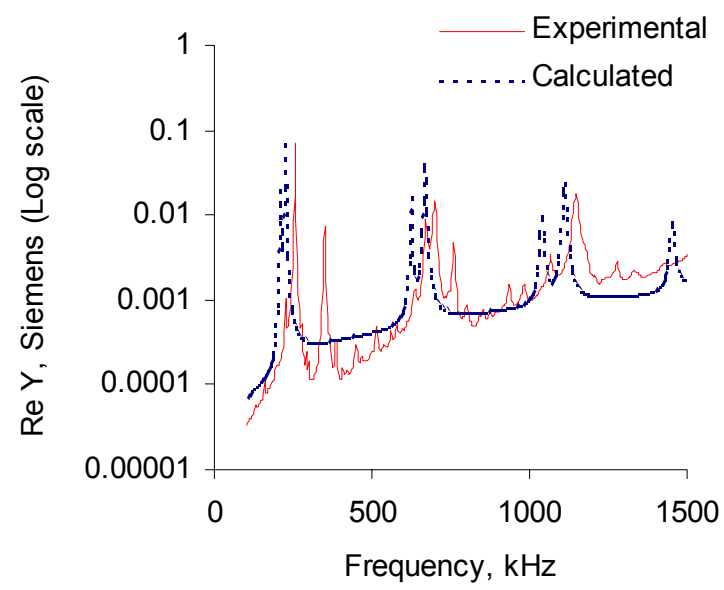

Figure 14 Experimental and calculated admittance spectra for square plate active sensor $(l=6.99 \mathrm{~mm}, b=6.56$ $\mathrm{mm}, \mathrm{t}=0.215 \mathrm{~mm}, \varepsilon_{33}^{T}=15.470 \cdot 10^{9} \mathrm{~F} / \mathrm{m}, s_{11}^{E}=15.3 \cdot 10^{-12} \cdot \mathrm{Pa}^{-1}$, $\left.d_{31}=-175 \cdot 10^{-12} \cdot \mathrm{m} / \mathrm{V}, \mathrm{k}_{31}=0.36\right)$.

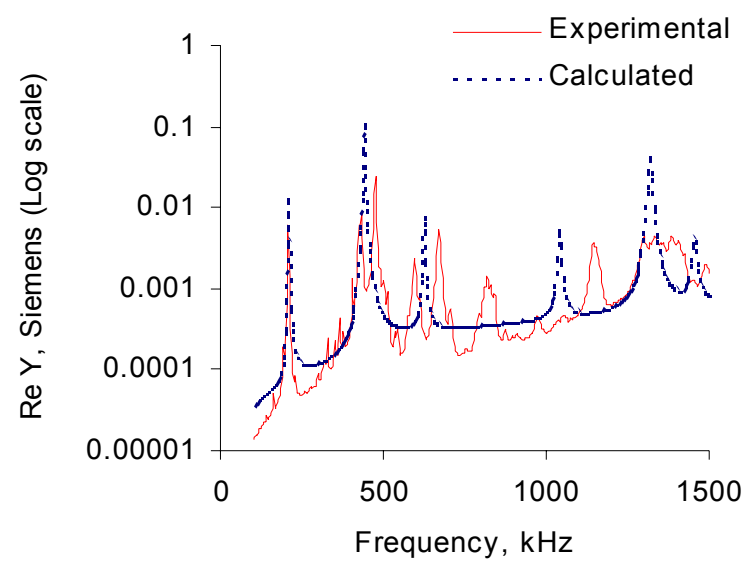

Figure15 Experimental and calculated admittance spectra for half-breadth active sensor $(\mathrm{l}=6.99 \mathrm{~mm}, \mathrm{~b}=3.53$ $\mathrm{mm}, \mathrm{t}=0.215 \mathrm{~mm}, \varepsilon_{33}^{T}=15.470 \cdot 10^{9} \mathrm{~F} / \mathrm{m}, s_{11}^{E}=15.3 \cdot 10^{-12} \cdot \mathrm{Pa}^{-1}$, $\left.d_{31}=-175 \cdot 10^{-12} \cdot \mathrm{m} / \mathrm{V}, \mathrm{k}_{31}=0.36\right)$.

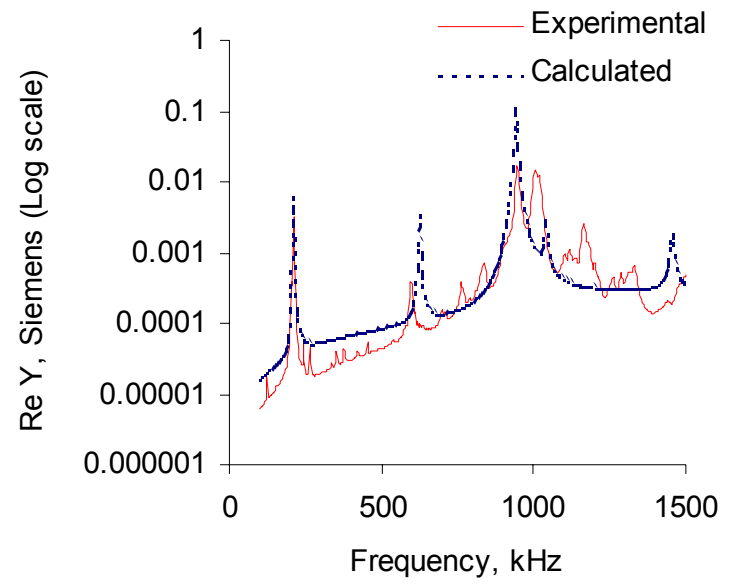

Figure 16 Experimental and calculated admittance spectra for quarter-breadth active sensor $(1=6.99 \mathrm{~mm}, \mathrm{~b}=$ $1.65 \mathrm{~mm}, \mathrm{t}=0.215 \mathrm{~mm}, \varepsilon_{33}^{T}=15.470 \cdot 10^{9} \mathrm{~F} / \mathrm{m}, s_{11}^{E}=15.3 \cdot 10^{-}$ $\left.{ }^{12} \cdot \mathrm{Pa}^{-1}, d_{31}=-175 \cdot 10^{-12} \cdot \mathrm{m} / \mathrm{V}, \mathrm{k}_{31}=0.36\right)$.

Table 5 Results of the dynamic characterization of one circular and three rectangular APC-850 piezoelectric wafers $(R=$ axisymmetric radial vibration; $L=$ in-plane length vibration; $B=$ in-plane breadth vibration; $T=$ out-of-plane thickness vibration)

\begin{tabular}{|c|c|c|c|c|c|c|c|c|c|c|}
\hline & & \multicolumn{9}{|c|}{ Frequency, $\mathrm{kHz}$} \\
\hline \multirow{3}{*}{ Circular wafer $6.98 \mathrm{~mm} \times 0.216-\mathrm{mm}$} & Exp. & \multirow{3}{*}{\multicolumn{2}{|c|}{$\begin{array}{c}300(1 \mathrm{R}) \\
303(1 \mathrm{R}) \\
-1.0 \%\end{array}$}} & \multirow{3}{*}{\multicolumn{2}{|c|}{$\begin{array}{l}784(2 \mathrm{R}) \\
796(2 \mathrm{R}) \\
-1.5 \%\end{array}$}} & \multirow{3}{*}{\multicolumn{2}{|c|}{$\begin{array}{l}1,247(3 R) \\
1267(3 R) \\
-1.6 \%\end{array}$}} & \multirow{3}{*}{\multicolumn{2}{|c|}{$\begin{array}{l}1,697(4 \mathrm{R}) \\
1,733(4 \mathrm{R}) \\
-2.1 \%\end{array}$}} & $10,895(1 \mathrm{~T})$ \\
\hline & Calc. & & & & & & & & & $10,690(1 \mathrm{~T})$ \\
\hline & Error & & & & & & & & & $1.9 \%$ \\
\hline \multirow{3}{*}{$\begin{array}{l}\text { Square wafer } \\
6.99-\mathrm{mm} \times 6.56-\mathrm{mm} \times 0.215-\mathrm{mm}\end{array}$} & Exp. & 257 & 352 & 670 & 702 & 1070 & 1150 & 1572 & 1608 & 10,565 \\
\hline & Calc. & $\begin{array}{c}207.6 \\
(1 \mathrm{~L})\end{array}$ & $\begin{array}{c}221.6 \\
(1 \mathrm{~B})\end{array}$ & $\begin{array}{l}623 \\
(2 L)\end{array}$ & $\begin{array}{l}665 \\
(2 B)\end{array}$ & $\begin{array}{c}1038 \\
(3 \mathrm{~L})\end{array}$ & $\begin{array}{l}1108 \\
(3 B)\end{array}$ & $\begin{array}{c}1453 \\
(4 \mathrm{~L})\end{array}$ & $\begin{array}{l}1551 \\
(4 \mathrm{~B})\end{array}$ & $\begin{array}{c}10,488 \\
(1 T)\end{array}$ \\
\hline & Error & $23.8 \%$ & $58.8 \%$ & $7.5 \%$ & $5.6 \%$ & $3.1 \%$ & $3.8 \%$ & $8.2 \%$ & $3.4 \%$ & $0.7 \%$ \\
\hline \multirow{3}{*}{$\begin{array}{l}1 / 2 \text { breadth wafer } \\
6.99-\mathrm{mm} \times 3.53-\mathrm{mm} \times 0.215-\mathrm{mm}\end{array}$} & Exp. & 208 & 432 & 597 & 670 & 821 & 1153 & 1307 & 1491 & 10,567 \\
\hline & Calc. & $\begin{array}{l}207.6 \\
(1 \mathrm{~L})\end{array}$ & $\begin{array}{l}439 \\
(1 \mathrm{~B})\end{array}$ & $\begin{array}{l}621 \\
(2 \mathrm{~L})\end{array}$ & & & $\begin{array}{l}1038 \\
(3 L)\end{array}$ & $\begin{array}{l}1318 \\
(2 \mathrm{~B})\end{array}$ & $\begin{array}{l}1451 \\
(4 L)\end{array}$ & $\begin{array}{c}10,488 \\
(1 T)\end{array}$ \\
\hline & Error & $0.2 \%$ & $-1.6 \%$ & $-4.0 \%$ & & & $11.1 \%$ & $\overline{-}$ & $2.8 \%$ & $0.7 \%$ \\
\hline \multirow{3}{*}{$\begin{array}{l}1 / 4 \text { breadth wafer } \\
6.99-\mathrm{mm} \times 1.64-\mathrm{mm} \times 0.215-\mathrm{mm}\end{array}$} & Exp. & 212 & & 597 & 950 & 1020 & & & 1496 & 10,905 \\
\hline & Calc. & $\begin{array}{c}207.6 \\
(1 \mathrm{~L})\end{array}$ & & $\begin{array}{l}621 \\
(2 \mathrm{~L})\end{array}$ & $\begin{array}{c}940 \\
(1 \mathrm{~B})\end{array}$ & $\begin{array}{c}1038 \\
(3 \mathrm{~L})\end{array}$ & & & $\begin{array}{c}1451 \\
(4 \mathrm{~L})\end{array}$ & $\begin{array}{c}10,488 \\
(1 \mathrm{~T})\end{array}$ \\
\hline & Error & $2.1 \%$ & & $-4.0 \%$ & $1.1 \%$ & $-1.8 \%$ & & & $3.1 \%$ & $1.0 \%$ \\
\hline
\end{tabular}


Figure 15 shows the admittance real-part spectrum for the half-breadth piezoelectric active sensor (aspect ratio $2: 1$ ). In the $0-1500 \mathrm{kHz}$ frequency band, the theoretical curve displays 6 peaks. The corresponding resonance frequencies (Table 5) are $208 \mathrm{kHz}(1 \mathrm{~L}), 621 \mathrm{kHz}(2 \mathrm{~L}), 1038$ $\mathrm{kHz}(3 \mathrm{~L}), 1451 \mathrm{kHz}(4 \mathrm{~L})$, for lengthwise vibration, and 439 $\mathrm{kHz}(1 \mathrm{~B}), 1318 \mathrm{kHz}(2 \mathrm{~B}) \mathrm{kHz}$ for breadthwise vibration. The experimental results agree very well for the $1 \mathrm{~L}$ and $1 \mathrm{~B}$ modes ( $<2 \%$ error), a little less for the $2 \mathrm{~L}$ and $2 \mathrm{~B}$ modes $(4$ $-5 \%$ error) and reasonable for $3 \mathrm{~L}$ mode $(10 \%)$. The agreement for the $4 \mathrm{~L}$ mode is also good. In addition to these clearly identifiable modes, several other peaks are present in the experimental curve. These modes are attributed to the edge roughness producing secondary vibration effects. We conclude that, although the half-breadth sensor (aspect ratio 2:1) is still far from a proper 1-D case, a clear trend towards mode separation and a definite improvement in the firstmode prediction accuracy can be observed.

Figure 16 shows the admittance real-part spectrum for the quarter-breadth piezoelectric active sensor (aspect ratio $4: 1$ ). In the $0-1500 \mathrm{kHz}$ frequency band, the theoretical curve displays six peaks. The corresponding resonance frequencies, as given in Table 5, are $208 \mathrm{kHz}(1 \mathrm{~L}), 621 \mathrm{kHz}$ (2L), $1038 \mathrm{kHz}(3 \mathrm{~L}), 1451 \mathrm{kHz}(4 \mathrm{~L})$, and $940 \mathrm{kHz}(1 \mathrm{~B})$ $\mathrm{kHz}$

The experimental results, as presented in Figure 14 and Table 5, are in good agreement with the theory, especially for the $1 \mathrm{~L}, 2 \mathrm{~L}, 3 \mathrm{~L}$, and $1 \mathrm{~B}$, resonances that stay within $4 \%$ error. The $4 \mathrm{~L}$ resonance shows a rather higher $(-9 \%)$ error. In addition, the experimental curve shows an additional peak at $1167 \mathrm{kHz}$, which does not have a theoretical explanation. The overall conclusion is that, for $4: 1$ aspect ratio, good agreement between experiment and 1-D theory is obtained.

As a final note on this section, a few comments about the allocation of the symbols R, L, B and T are being made. In Table 5, these symbols were used to signify the natural frequencies calculated for axisymmetric radial, in-plane length-wise, in-plane breadth-wise, and out-of-plane thickness-wise vibrations. Then, the measured frequencies were allocated based on their numerical proximity to a certain theoretical frequency. Admittedly, this approach could introduce uncertainties regarding modes allocation, if mode clusters were present. Fortunately, most of the modes were well-separated, permitting a straightforward mode allocation. However, in a few instances, uncertainty in mode allocation was encountered (e.g., in some of the $1 / 1$ and $1 / 2$ rectangular plates results). To eliminate this uncertainty, further investigation could be done using a laser vibrometer to determine the actual vibration mode shapes. Such investigations are planned as follow up studies. Beam and plate Experiments To verify the applicability of Equation (33) and better understand the relationship between structural resonances and the active-sensor response, experiments on simple beam and plate specimens were performed. The experimental results were compared with theoretical prediction, and method's salient features were highlighted.

\section{Beam Experiments}

One-dimensional beam structures are easy to model, and the prediction of their natural frequencies is fairly well understood (Inman, 1996). A number of small steel beams of various thickness and breadth values were fabricated (Figure 17). All specimens were instrumented with 7-mm square PZT active sensors placed at $40 \mathrm{~mm}$ from one end. Standard sensor-installation procedure was employed. During the experiments, the specimens were supported on commercially available packing foam to simulate free-free conditions. These small beams, ranging in weight from 16.4 $\mathrm{g}$ to $82 \mathrm{~g}$, were chosen as representative of precision machinery parts that are the candidate application for this embedded-sensor structural identification technology. During the experiments, the E/M impedance and admittance spectra were recorded in a wide frequency range. When necessary, frequency zoom was employed. Theoretical analysis was performed for free-free boundary conditions, and the corresponding frequencies and mode shapes were derived using Equations (46) and (47). Prediction of E/M impedance and admittance response was performed using Equations (33) and (45). The beam natural frequencies were also identified from the E/M impedance real part spectrum. As shown by Giurgiutiu and Zagrai (2001), these frequencies could be identical with the basic beam resonances, as predicted by classical vibration analysis (Inman, 1996). It was also shown that when the sensor dynamics and structural dynamics are widely separated, the sensor's own dynamics can be neglected and the quasi-static approximation for sensor stiffness, as depicted in Equation (1), can be utilized.

In our case, the sensor first frequency was around 208 $\mathrm{kHz}$, while the structural frequency range was $0-30 \mathrm{kHz}$. In this case, no distinguishable difference could be found between the use of fully dynamic sensor model, and the quasi-static sensor model. However, at higher frequencies, when the sensor and structural dynamics start to interfere, the use of fully dynamic sensor model is warranted. When the beam thickness and breadth were varied, the frequencies changed accordingly. As an example, Figure 18 shows the calculated and measured E/M impedance real-part spectra for beam \#1 of Figure 18 (to fit into same graph, selective scaling of the theoretical and measure curves was applied). In this $0-30 \mathrm{kHz}$ range, several flexural modes (2F @ $3.850 \mathrm{kHz}, 3 \mathrm{~F} @ 7.547 \mathrm{kHz}, 4 \mathrm{~F} @ 12.475 \mathrm{kHz}, 5 \mathrm{~F} @$ $18.635 \mathrm{kHz})$ and one axial mode $(1 \mathrm{~A} 26.035 \mathrm{kHz})$ are clearly visible. Not easily identifiable on Figure 18 is the first flexural mode (1F @ $1.396 \mathrm{kHz}$ ), which is overshadowed by the dominant $1 / \omega C$ behavior of the impedance curve at low frequencies. As a general note, we mention that embedded active sensors techniques are more 
suitable for high frequency modal identification, where the

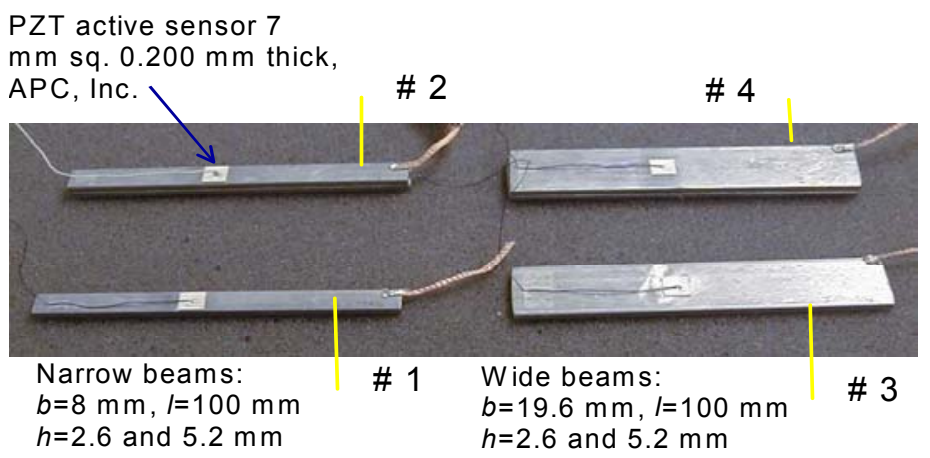

Figure 17 Experimental specimens to simulate one-dimensional structure.
$1 / \omega C$ effect is much diminished.

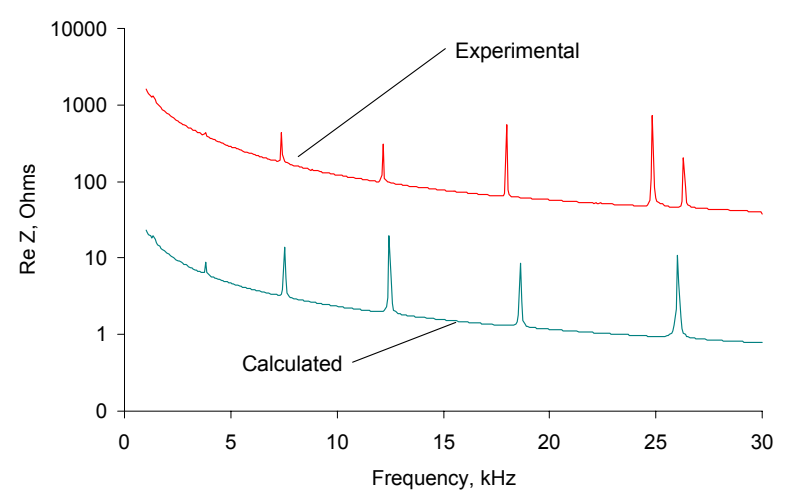

Figure 18 Experimental and calculated spectra of frequencies for beam \#1 of Figure 17.

the structural substrate. The structural dynamics was incorporated through the pointwise dynamic stiffness presented by the structure to the sensor. The model accounts for both axial and flexural vibrations and predicts the electro-mechanical (E/M) impedance response, as it would be measured at the piezoelectric active sensor's terminals. The derived mathematical expressions accounted for the dynamic response of both the sensor and the structure. This feature is especially important for proper modeling of the sensor-structure interaction at the high end of the structural frequencies spectrum. In support of the theoretical investigation, experiments conducted on simple specimens were performed. The performed experiments were able to correctly identify structural resonance in the $0-30 \mathrm{kHz}$ range and beyond. It was shown that $\mathrm{E} / \mathrm{M}$ impedance spectrum recorded by the piezoelectric active sensor reflects the mechanical response of the structure around the structural natural frequencies. Our studies have shown that, when attached to the structure, these thin and small PZT wafers would not detectably modify the structural dynamics.

In our experiments, special attention was given to investigating sensor's consistency and repeatability. Statistical sensor batches were used and statistical analysis was performed. It was found that PZT active sensors have good and stable characteristics not only in the as-received state, but also when mounted on a structure.

A limitation of using PZT wafer embedded active sensors for structural identification is that these sensors, which are tuned to high frequency explorations, do not behave well below $5 \mathrm{kHz}$. This is due to the overshadowing effect of the $1 / \omega C$ characteristics, which becomes very strong at low frequencies. In the $1-5 \mathrm{kHz}$ range, we were able to alleviate these difficulties by narrowband tuning. However, below $1 \mathrm{kHz}$, the method is not recommended.

In view of advantages and disadvantages, it is felt that piezoelectric active sensors in conjunction with the $\mathrm{E} / \mathrm{M}$ 
impedance technique, have their niche as a structural identification methodology using self-sensing, permanently attached active sensors. They can be confidently used at high frequencies where other embedded sensors (e.g., accelerometers and strain gauges) usually encounter bandwidth difficulties. Due to their perceived low cost, these active sensors can also be inexpensively configured as sensor arrays. The proposed method can be a useful and reliable tool for automatic on-line structural identification in the ultrasonic frequencies range. The use of piezoelectricwafer active sensors can be not only advantageous, but, in certain situations, may be the sole investigative option, as in the case of precision machinery, small but critical turbomachinery parts, and computer industry components.

\section{ACKNOWLEDGMENTS}

The financial support of Department of Energy through the Sandia National Laboratories, contract doc. \# BF 0133 is thankfully acknowledged. Sandia National Laboratories is a multi-program laboratory operated by Sandia Corporation, a Lockheed Martin Company, for the United States Department of Energy under contract DE-AC0494AL85000.

\section{REFERENCES}

Ayres, T., Chaudhry Z., and Rogers C., (1996) "Localized Health Monitoring of Civil Infrastructure via Piezoelectric Actuator/Sensor Patches," Proceedings, SPIE's 1996 Symposium on Smart Structures and Integrated systems, SPIE Vol. 2719, pp. 123-131.

Banks, H. T., Smith, R. C., Wang, Y., (1996) "Smart Material Structures: Modeling, Estimation and Control”, Masson, John Wiley \& Sons, Paris 1996.

Blevins, R. D., (1979) "Formulas for Natural Frequency and Mode Shape", Litton Educational Publishing Inc., 1979.

Chaudhry, Z., Sun, F. P, and Rogers C. A., (1994) "Health Monitoring of Space Structures Using Impedance Measurements," Fifth International Conference on Adaptive Structures, Sendai, Japan, 5-7 December, 1994; pp. 584-591.

Chaudhry, Z., Joseph, T., Sun, F., and Rogers, C., (1995). "Local-Area Health Monitoring of Aircraft via Piezoelectric Actuator/Sensor Patches," Proceedings, SPIE North American Conference on Smart Structures and Materials, San Diego, CA, 26 Feb. - 3 March, 1995; Vol. 2443, pp. 268-276.

Clark, R. L., Burdisso, R A., Fuller, C. R., (1993) "Design approaches for shaping polyvinylidene fluoride sensors in active structural acoustic control" Journal of Intelligent Material Systems and Structures, v 4 n 3 Jul 1993 p 354-365, 1993.

Collins, K., Plaut, R., and Wauer, J., (1992) "Free and Forced Longitudinal Vibrations of Cantilevered Bar with a Crack", Journal Vibration Acoustics Stress Reliability Design, 114, pp.171-177, 1992.

Crawley, E. A. and deLuis, J., (1987) "Use of Piezoelectric Actuators as Elements of Intelligent Structures”, AIAA Journal, Vol. 25, No. 10, pp. 1375-1385, 1987.

D'Cruz, J., (1993) "Active control of panel vibrations with piezoelectric actuators", Journal of Intelligent Material Systems and Structures, v 4 n 3 Jul 1993 p 398-402, 1993.

Dimitriadis, E. K., Fuller, C. R., Rogers, C. A., (1991) "Piezoelectric actuators for distributed vibration excitation of thin plates", Vol.113, Journal of Vibration and Acoustics, 1991.

Esteban, J., (1996) "Analysis of the Sensing Region of a PZT ActuatorSensor", Ph. D. Dissertation, Virginia Polytechnic Institute and State University, July 1996.

Ewins, D. J., (1984) "Modal Test: Theory and Practice”, Research Studies
Press Ltd., Letchworth, Hertfortshire, England, 1984.

Giurgiutiu, V., Chaudhry, Z., Rogers, C.A., (1994) "The Analysis of Power Delivery Capability of Induced Strain Actuators for Dynamic Applications", Proceedings of the Second International Conference on Intelligent Materials, ICIM'94, June 5-8, 1994, Colonial Williamsburg, VA, Technomic Pub. Co., Inc., 1994, p.p. 565-576.

Giurgiutiu, V., Reynolds, A., and Rogers, C. A. (1999) "Experimental Investigation of E/M Impedance Health Monitoring of Spot-Welded Structural Joints" Journal of Intelligent Material Systems and Structures, Vol. 10, No. 10, October 1999, pp. 802-811.

Giurgiutiu, V.; Zagrai, A. (2000) "Damage Detection in Simulated AgingAircraft Panels Using the Electro-Mechanical Impedance Technique", Adaptive Structure and Material Systems Symposium, ASME Winter Annual Meeting, Nov. 5-10, 2000, Orlando, FL.

Giurgiutiu, V.; Zagrai, A. (2001) "Embedded Self-Sensing Piezoelectric Active Sensors for On-Line Structural Identification", Submitted to: Transactions of ASME, Journal of Vibration and Acoustics, January 2001.

Graff, K., F., (1975) "Wave Motion in Elastic Solids", Dover Publications, Inc., 1991

Harris, C., M., (1996) "Shock and Vibration Handbook", McGraw-Hill, USA, 1996.

IEEE Std. 176 (1987), IEEE Standard on Piezoelectricity, The Institute of Electrical and Electronics Engineers, Inc., 1987.

Ikeda, T., (1996), "Fundamentals of Piezoelectricity", Oxford science publications, 1996.

Inman, D. J., (1996) “Engineering Vibration”, Prentice-Hall, Inc., 1996.

Kelly, S. G., (2000) "Fundamentals of Mechanical Vibration", $2^{\text {nd }}$ edition , McGraw-Hill, 2000.

Lazan, B. J. (1968) Damping of Materials and Members in Structural Mechanics, Pergamon Press, 1968.

Liang, C., Sun, F. P., and Rogers C. A., (1994) "Coupled electroMechanical Analysis of Adaptive Material System-Determination of the Actuator Power Consumption and System energy Transfer", Journal of Intelligent Material Systems and Structures, Vol. 5, January 1994, pp. 12-20

Meirovitch, L., (1986) "Elements of Vibration Analysis", $2^{\text {nd }}$ edition, McGraw-Hill, 1986.

Onoe, M.; Jumonji, H. (1967) "Useful formulas for piezoelectric ceramic resonators and their application to Measurement of parameters", IRE, Number 4, Part 2, 1967

Park, G., Cudney, H. H., Inman, D. J., (2000) “An Integrated health monitoring technique using structural Impedance sensors" Journal of Intelligent Material Systems and Structures, Vol. 11, No. 6, June 2000, pp. 448-455.

Parton, V.Z., Kudryavtsev (1988) "Electromagnetoelasticity, Piezoelectrics and Electrically Conductive Solids", Gordon and Breach science publishers, 1988

Pugachev S. I., Ganopolsky V. V., Kasatkin B. A., Legysha F. F, Prydko N. I. (1984) "Piezoceramic transducers", Handbook , Sudostroenie, St.Petersburg, 1984, (in Russian).

Sun, F. P., Liang C., and Rogers, C. A., (1994) "Experimental Modal Testing Using Piezoceramic Patches as Collocated Sensors-Actuators", Proceeding of the 1994 SEM Spring Conference \& Exhibits, Baltimore, MI, June 6-8, 1994.

Timoshenko, S., P. (1955) "Vibration problems in Engineering", D.Van Nostrand Company Inc., 1955.

Waanders, J. W. (1991) "Piezoelectric Ceramic, Properties and Applications", Philips Components, Eindhoven, Netherlands, 1991.

Wang, B., Chen, R., (2000) "The Use of Piezoceramic Transducers for Smart Structural Testing", Proceeding of SPIE 2000 Conference, Newport Beach, CA.

Wang, B., Rogers, C.A., (1991) "Modeling of finite-length spatiallydistributed induced strain actuators for laminate beams and plates", In Procceding, AIAA/ASME/ASCE/AHS/ASC 32 ${ }^{\text {nd }}$ Structures, Structural Dynamics, and Material Conference, 1991.

Zhou, S., Liang, C., and Rogers, C., (1996) "An Impedance-Based System Modelling approach for Induced Strain Actuator-Driven Structures", Journal of Vibration and Acoustics, July 1996, pp.323-331. 